\title{
THE STABILITY OF THE POINT-SPREAD FUNCTION OF THE ADVANCED CAMERA FOR SURVEYS ON THE HUBBLE SPACE TELESCOPE AND IMPLICATIONS FOR WEAK GRAVITATIONAL LENSING ${ }^{1}$
}

\author{
Jason D. Rhodes, ${ }^{2,3}$ Richard J. Massey, ${ }^{3}$ Justin Albert, ${ }^{3}$ Nicholas Collins, ${ }^{4}$ Richard S. Ellis, ${ }^{3}$ \\ Catherine Heymans, ${ }^{5}$ Jonathan P. Gardner, ${ }^{4} \mathrm{Jean}$ Paul Kneib, ${ }^{6}$ Anton Koekemoer, ${ }^{7}$ \\ Alexie Leauthaud, ${ }^{6}$ Yannick Mellier, ${ }^{8,9}$ Alexander Refregier, ${ }^{10}$ \\ JAMES E. TAYLOR, ${ }^{3,11}$ AND LudOVIC VAN WaERBEKE ${ }^{5}$ \\ Received 2006 September 21; accepted 2007 January 10
}

\begin{abstract}
We examine the spatial and temporal stability of the Hubble Space Telescope's Advanced Camera for Surveys (ACS) Wide Field Camera (WFC) point-spread function (PSF) using the $2 \mathrm{deg}^{2}$ COSMOS survey. This is important for studies of weak gravitational lensing, where the ability to deconvolve the PSF from galaxy shapes is of paramount importance. We show that stochastic aliasing of the PSF necessarily occurs during "drizzling." This aliasing is maximal if the output-pixel scale is equal to the input-pixel scale. This source of PSF variation can be significantly reduced by choosing a Gaussian drizzle kernel with a size of 0.8 input pixels and by reducing the output-pixel scale. We show that the PSF is temporally unstable, resulting in an overall slow periodic focus change in the COSMOS images. Using a modified version of the Tiny Tim PSF modeling software, we create grids of undistorted stars over a range of telescope focus values. We then use the approximately 10 well-measured stars in each COSMOS field to pick the best-fit focus value for each field. The Tiny Tim model stars can then be used to perform PSF corrections for weak lensing. We derive a parametric correction for the effect of charge transfer efficiency (CTE) degradation on the shapes of objects in the COSMOS field as a function of observation date, position within the ACS WFC field, and object flux. Finally, we discuss future plans to improve the CTE correction.
\end{abstract}

Subject headings: instrumentation: detectors — surveys — techniques: image processing

Online material: color figures

\section{INTRODUCTION}

The addition of the Advanced Camera for Surveys (ACS; Pavlovsky et al. 2006) to the Hubble Space Telescope (HST) in 2002 February enabled significant science returns in a variety of disciplines due to the increased resolution, areal coverage, and quantum efficiency of the ACS Wide Field Camera (WFC) as compared to previous HST imaging instruments, including WFPC, WFPC2, and STIS. Despite the myriad successes of ACS, some areas of study have been hampered by a lack of understanding of the properties of the ACS WFC point-spread function (PSF). In particular, studies of weak gravitational lensing, in which the shapes of background galaxies undergo a small coherent distortion by foreground dark matter, are made difficult by time variability of the ACS PSF. Since we are interested in weak gravitational lens-

\footnotetext{
1 Based on observations with the NASA/ESA Hubble Space Telescope, obtained at the Space Telescope Science Institute, which is operated by the Association of Universities for Research in Astronomy, Inc., under NASA contract NAS5-26555; also based on data collected at the Subaru Telescope, which is operated by the National Astronomical Observatory of Japan; the XMM-Newton, an ESA science mission with instruments and contributions directly funded by ESA member states and NASA; the European Southern Observatory, Chile; Kitt Peak National Observatory, Cerro Tololo Inter-American Observatory, and the National Optical Astronomy Observatory, which is operated by the Association of Universities for Research in Astronomy, Inc., under cooperative agreement with the National Science Foundation; the National Radio Astronomy Observatory, which is a facility of the National Science Foundation operated under cooperative agreement by Associated Universities, Inc.; and the Canada-France-Hawaii Telescope, operated by the National Research Council of Canada, the Centre National de la Recherche Scientifique de France, and the University of Hawaii.

2 Jet Propulsion Laboratory, Pasadena, CA 91109.

3 California Institute of Technology, MC 105-24, 1200 East California Boulevard, Pasadena, CA 91125.
}

ing, we concentrate our studies on the ACS WFC and do not study the PSF of other ACS channels. Hereafter, when we refer to the ACS we are referring to the ACS WFC. Because the slight shape distortions due to weak lensing are up to an order of magnitude smaller than the shape distortions of small galaxies due to the ACS PSF, it is critical to have accurate PSF models for use in deconvolving the galaxy shapes from the PSF. There are notable exceptions in which ACS has had great success in weak lensing, including the study of weak lensing by galaxy clusters (e.g., Lombardi et al. 2005; Jee et al. 2005, 2006), and the results of Heymans et al. (2005) using the GEMS survey. In the case of lensing by galaxy clusters, the weak-lensing signal is typically large compared to the signal arising from large-scale structure alone, and thus PSF effects are less important. For clusters at $z>1$, PSF effects become important, and Jee et al. (2006) have found that a limited number of PSF models derived from globular cluster observations can be used for PSF correction. The

\footnotetext{
${ }^{4}$ Exploration of the Universe Division, Observational Cosmology Laboratory, Code 665, Goddard Space Flight Center, Greenbelt, MD 20771.

5 University of British Columbia, 6224 Agricultural Road, Vancouver, BC V6T 1Z1, Canada.

${ }^{6}$ Laboratoire d'Astrophysique de Marseille, BP 8, Traverse du Siphon, 13376 Marseille Cedex 12, France.

${ }^{7}$ Space Telescope Science Institute, 3700 San Martin Drive, Baltimore, MD 21218 .

${ }^{8}$ Institut d'Astrophysique de Paris, UMR7095 CNRS, Université Pierre \& Marie Curie - Paris, 98 bis Boulevard Arago, 75014 Paris, France.

${ }^{9}$ Observatoire de Paris, LERMA, 61, avenue de l'Observatoire, 75014 Paris, France.

${ }^{10}$ Service d'Astrophysique, CEA/Saclay, 91191 Gif-sur-Yvette, France.

${ }_{11}$ Department of Physics and Astronomy, University of Waterloo, Waterloo, QC, Canada.
} 
GEMS survey contained many images taken in a relatively short amount of time, such that the PSF time variations could be internally calibrated using stars in the survey. However, for surveys taken over an extended period of time in which there are few stars and the weak-lensing signal is low, the time variability of the ACS PSF precludes using the stars in each exposure to make a PSF model suitable for weak-lensing PSF deconvolution. In this paper we offer an alternative solution in which templates of model PSFs are created and the few available stars in each ACS field are used to select the appropriate PSF template to be used for PSF deconvolution. A similar method using templates created by combining the stars from many exposures with similar PSF properties was used by Schrabback et al. (2006). Another alternative approach is to use globular cluster fields with many stars to model the PSF (e.g., Jee et al. 2005, 2006). In addition to the temporally varying telescope PSF, degradation of the charge transfer efficiency (CTE) of the ACS CCDs is gradually adding additional variation to the measured PSF. The GEMS survey, taken relatively early in the life of the ACS, did not necessitate a correction for this effect, but more recent surveys do need to take this effect into account. This paper also addresses that problem by providing a prescription by which measured galaxy shapes can be corrected for the effects of CTE degradation.

The Cosmic Evolution Survey (COSMOS) $2 \mathrm{deg}^{2}$ field ( $\sim 1.64 \mathrm{deg}^{2}$ with the ACS; Scoville et al. 2007) has a unique combination of area, depth, and resolution that opens up new areas of study with the HST. This is particularly true in weak lensing, where the surface density of resolved galaxies (up to $\sim 80 \mathrm{arcmin}^{-2}$ ) is significantly higher than that available from ground-based surveys (10-30 $\mathrm{arcmin}^{-2}$ ). Along with the excellent photometric redshifts available in the COSMOS field due to extensive ground-based follow-up, this allows studies of cosmic shear tomography at small angular scales to unprecedented accuracy (Massey et al. 2007a). COSMOS also uniquely affords the opportunity for highresolution dark matter maps over a wide field (Massey et al. 2007c). Such analyses depend critically on the ability to deconvolve the effects of the PSF from the measured galaxy shapes, and we demonstrate in this paper a procedure for doing so. The demanding nature of our weak-lensing analysis will provide PSF models of immediate applicability to other studies, including an examination of AGNs in the COSMOS field (J. Gabor et al. 2007 , in preparation).

Typical ground-based weak-lensing surveys use the stars within each field to make a model of the PSF. This PSF model, often represented as a polynomial across the field, is then used to deconvolve the PSF from galaxy shapes using a variety of methods (see Heymans et al. [2006] and Massey et al. [2007b] for a description of many of these methods). The modeling and deconvolution of the PSF remains the primary systematic for weaklensing studies, and considerable work is currently going into optimizing the methods for doing this (again, see Heymans et al. 2006). HST images useful for weak lensing contain too few stars in each image to make a viable PSF model. In the past, this has been overcome by collecting stars from many images to model the PSF (e.g., Rhodes et al. 2001; Hoekstra et al. 1998). However, we find that the ACS PSF is not sufficiently stable to allow for the collection of stars from many images, which would provide models at the accuracy level needed for cosmic shear. Thus, we have modified the PSF creation software Tiny Tim (Krist \& Hook 2004) to create simulated PSFs that we use for PSF correction. The reasons the ACS PSF is more temporally unstable than that of previous cameras such as WFPC2 are twofold. First, WFPC2 is on-axis, while ACS is significantly off-axis, yielding a more sensitive measure of defocus. Second, ACS has a more asymmetric distortion compared to the largely radially symmetric pattern for WFPC2. These effects combine to provide more sensitivity to focus changes.

The degradation of CTE is caused by charge traps created by charged particle radiation damage-induced CCD defects (Riess \& Mack 2004; Mutchler \& Sirianni 2005). These traps cause a trailing of the charge during readout that can mimic a weak-lensing signal. This effect is particularly insidious for weak-lensing applications because it preferentially affects low-flux objects (such as galaxies) rather than high-flux objects (such as bright stars). So, the typical weak-lensing method of using high signal-to-noise ratio $(\mathrm{S} / \mathrm{N})$ stars to correct the PSF of low-S/N galaxies completely fails in the case of CTE degradation. Nor, strictly speaking, does it act as a convolution, although it has been treated as such to first order in the past (e.g., Rhodes et al. 2004). The effect of CTE degradation depends in a complicated way on the date of observation, the position within the $\mathrm{CCD}$, the flux of a source, and the background level of the image. The optimal solution to the problems caused by CTE degradation would be to understand and remove the effects of CTE on the images themselves as the first step in image reduction. However, the effects of CTE on individual pixel values have not yet been fully quantified and will be investigated in a future paper. We correct for CTE effects by subtracting a model of the spurious (CTE-induced) signal from the measured galaxy ellipticities at the end of our weak-lensing pipeline.

This paper is organized as follows. Section 2 describes the data and software tools we use to analyze the data. This section includes descriptions of the COSMOS survey and auxiliary data sets we use in $\S 2.1$, a brief description of the MultiDrizzle image reduction pipeline in $\S 2.2$, a description of the Tiny Tim PSF simulation software in $\S 2.3$, and a description of our weaklensing PSF-correction scheme in $\S 2.4$. We detail the imagereduction procedures we use for weak-lensing images in $\S 3$. In $\S 4$ we describe the Tiny Tim-based PSF models we have created, and the procedure we use to deconvolve the PSF from galaxy shapes is given in $\S 5$. We conclude in $\S 6$, where we describe how the methods we have detailed can be of use on other data sets and with other weak-lensing methods. In the conclusion we describe steps we have taken to make our PSF modeling software available to the community for use on other HST data sets.

\section{DATA AND SOFTWARE}

\subsection{Data}

The primary motivation for our analysis of the ACS PSF is to perform various weak gravitational lensing analyses on the COSMOS field. The COSMOS field is a contiguous square covering $1.637 \mathrm{deg}^{2}$ centered at R.A. $=10^{\mathrm{h}} 00^{\mathrm{m}} 28.6^{\mathrm{s}}$, decl. $=$ $+02^{\circ} 12^{\prime} 21.0^{\prime \prime}$ (J2000.0). COSMOS was imaged with the ACS WFC during HST Cycles 12 (proposal ID 9822) and 13 (proposal ID 10092), between 2003 October and 2005 June. This data set contains 575 slightly overlapping pointings, each with four individual exposures of $507 \mathrm{~s}$ dithered by $(5,60)$ pixels. At this depth, each $10 \mathrm{arcmin}^{2}$ COSMOS pointing contains approximately 800 galaxies useful for weak lensing (with sufficient size and $\mathrm{S} / \mathrm{N}$ ) and approximately 10 stars suitable for measuring the PSF. For a more complete description of the COSMOS field, see Scoville et al. (2007). COSMOS was imaged in the F814W (I) filter, and our measurements of the PSF are specific to that filter. However, the methods we have developed are general and can be applied to other ACS WFC filters using the software we have made public (see $\S 6$ ). The applicability of our software and methods is limited 

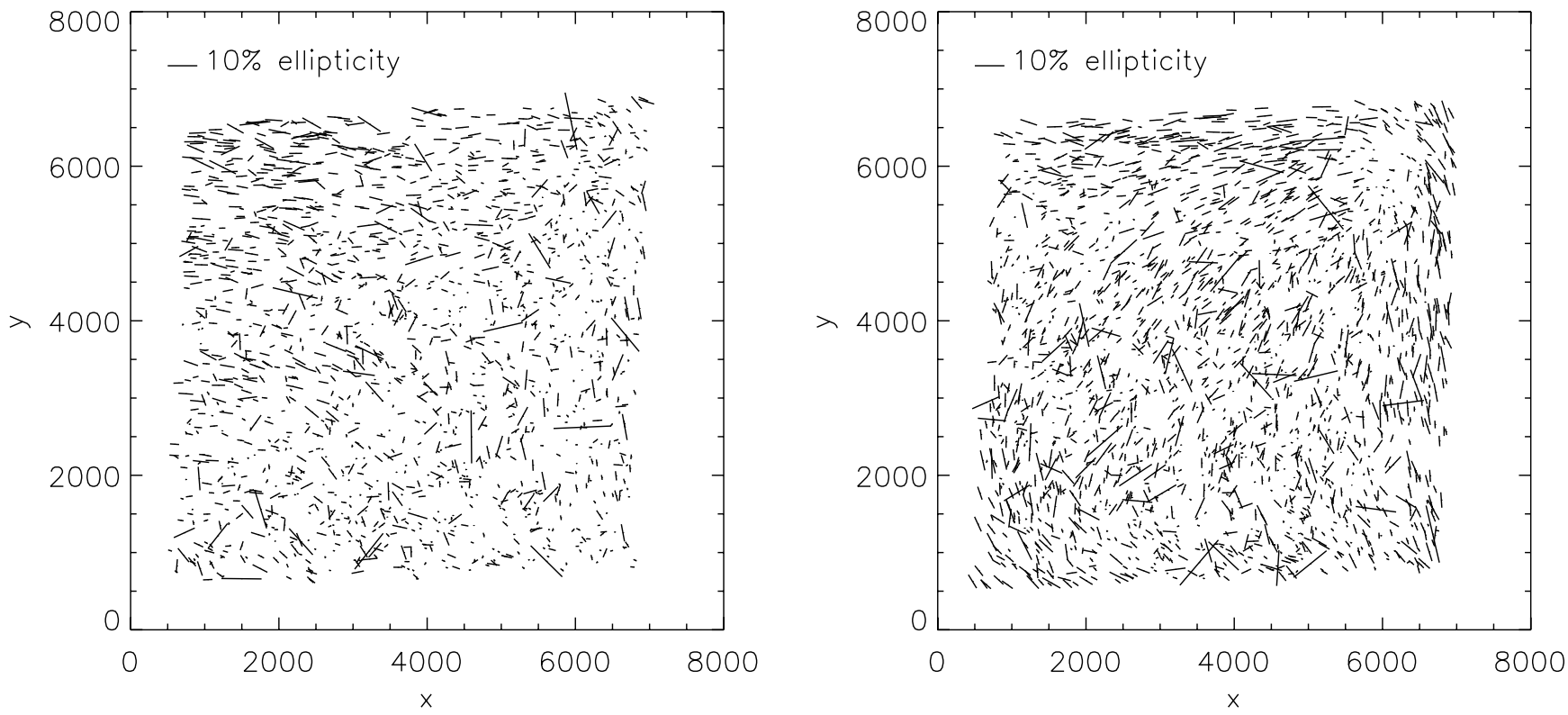

FIG. 1.- These figures demonstrate that the ACS PSF is time variable. Each tick mark represents the magnitude and direction of the ellipticity of a star in the COSMOS field. The left panel shows stars for the HST near the "nominal" focus value, and the right panel shows stars in images taken when the primary-to-secondary mirror separation was $3 \mu$ m closer than nominal. Since the focus varies with time, the PSF is not temporally stable. While the plots are noisy, the different underlying patterns can be clearly discerned.

by the ability of our modeling software, which does not include such effects as the long-wavelength (e.g., red) scattering within the ACS WFC CCDs (see, for instance, Sirianni et al. 2005).

The ACS WFC's PSF varies due to focus changes caused by thermal fluctuations of the HST on orbit (Heymans et al. 2005; Rhodes et al. 2006; Schrabback et al. 2006; Lallo et al. 2005, 2006; Makidon et al. 2006a, 2006b). This manifests itself in PSF patterns that vary significantly over time (Fig. 1). Since the changes in focus are stochastic, the focus of a particular observation cannot be predicted a priori and must be inferred from the data. We have developed a method for determining the telescope focus (see $\S 4$ ), and we show in Figure 2 the focus of the HST as a function of time over the course of the COSMOS observations. There are two timescales over which the HST focus changes. The first timescale is the 90 minute $H S T$ orbit; the telescope expands and contracts, or "breathes," as the telescope goes into and out of sunlight (see, for instance, Lallo et al. 2006). Since each COSMOS observation takes place over a single orbit, we average over this source of focus variation in COSMOS (and indeed in most HST applications). This intraorbit breathing is a large source of the individual error bars on the points in Figure 2, because the intraorbit focus changes are of order a few microns or more (Lallo et al. 2006). There is also an apparent slow oscillatory drift in the focus of the telescope over several weeks; it is this gradual drift that we attempt to model and correct for as described below. From Figure 2 it is clear that sampling the focus every month as done by Lallo
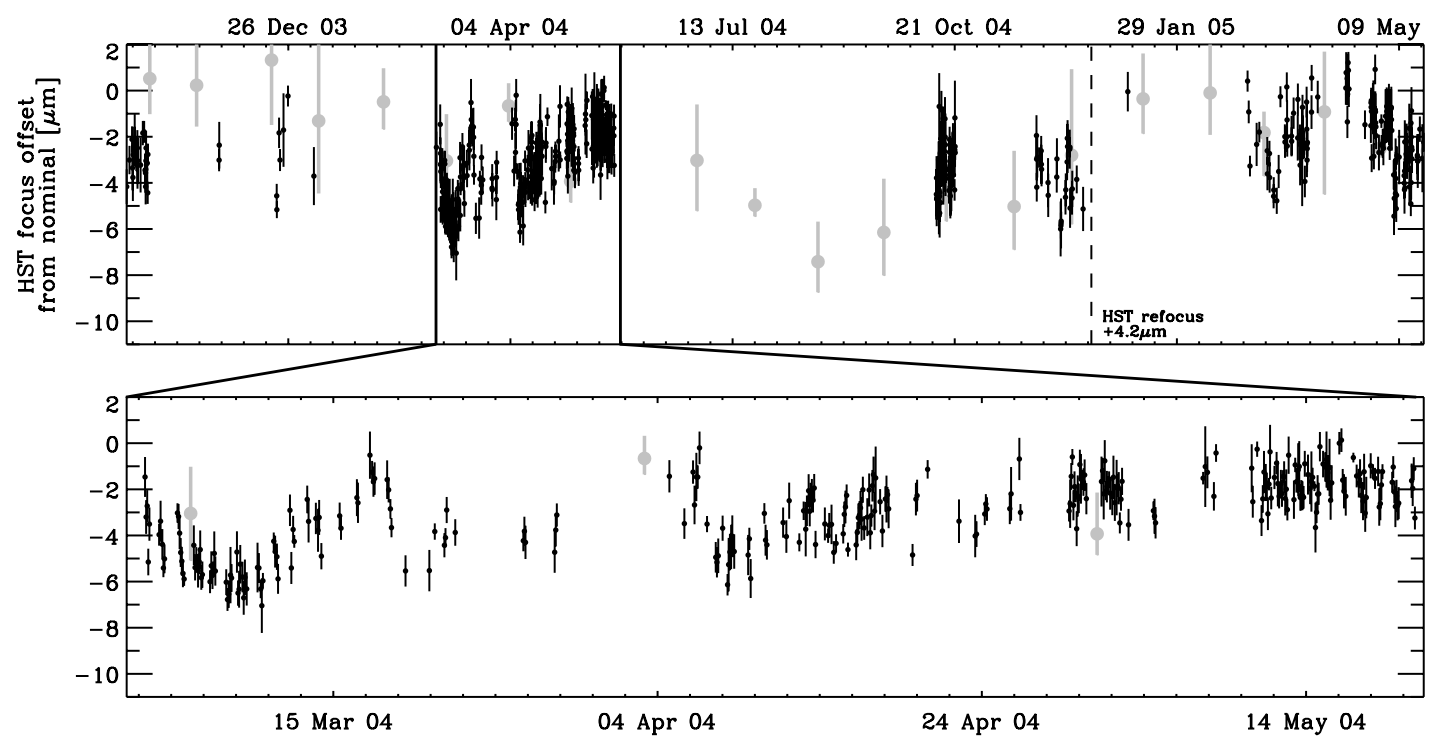

FIG. 2.-Top: Focus of HST for each field in the COSMOS survey (small data points). The errors bars are the error on the mean focus determined from the $\sim 10$ useful stars in each COSMOS image. The larger, fainter data points are focus values determined from a monitoring program using the ACS HRC (Lallo et al. 2006). Their error bars represent the full focus variation during an HST orbit. The date of the 2004 secondary mirror movement is labeled. Bottom: Focus for a small time window during Cycle 12 (2004 March to May). The cyclical pattern of the focus is clear. We describe how we determine the telescope focus value in $\S 4$. [See the electronic edition of the Journal for a color version of this figure.] 


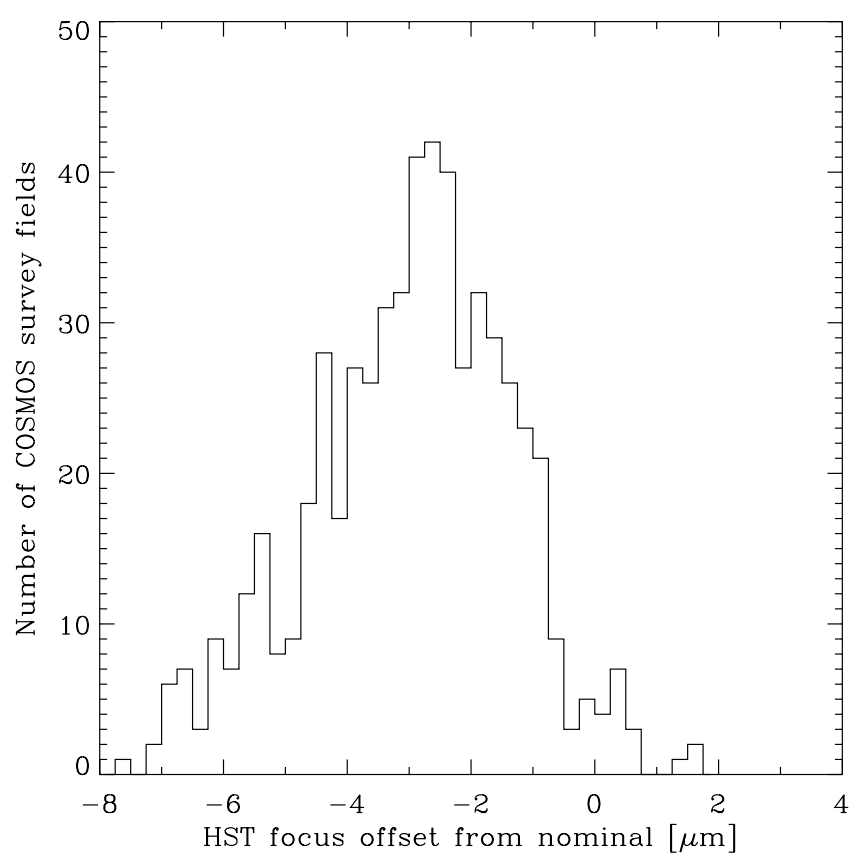

FIG. 3.- Histogram of the best-fit focus values for each of the 575 COSMOS fields.

et al. provides insufficient sampling to satisfactorily model this drift. We find that the focus is typically within $\pm 2 \mu \mathrm{m}$ of $-3 \mu \mathrm{m}$, as shown in Figure 3. During the first decade of HST operations it was necessary to periodically (approximately every 6 months) reset the telescope focus as the struts holding the secondary mirror in place slowly outgassed water and shrank. However, the last commanded focus changes of HST were in 2002 December, 2004 December, and 2006 July, because this slow focus drift due to the shrinking of the structure has stabilized.

In order to examine the behavior of the ACS PSF we make use of two other HST data sets. The first consists of images of the stellar field 47 Tuc taken as part of the ACS calibration program (GO-10048; Krist 2003). The three 47 Tuc images are single $30 \mathrm{~s}$ exposures of dense stellar fields taken between 2003 November 28 and 2004 September 7 with the F814W filter. These images are processed with the same pipeline we describe in $\S 3$, and we use these images to test our PSF models and focus prediction methods as described in $\S 4$.

We also use extended pixel edge response (EPER) data from $H S T$ calibration program 10369, consisting of images acquired using internal targets (lamps) only, taken during Earth occultation time (Mutchler \& Sirianni 2005). These images overscan the ACS CCD fiducial range of $2 \times 2048 \times 4096$ pixels, providing additional "virtual pixels" along the edges of the chips, into which pixel charge (but not actual signal from the lamps) can transfer during readout. The signal that occurs in the overscan region is charge that is released from charge traps during the readout process. This allows calibration of the CTE of the ACS CCDs. The EPER images are $1.2 \mathrm{~s}$ exposures, taken using a combination of the F555W and F435W filters to reduce the incident power from the lamps. Note that the CTE occurs within the CCDs, and so is completely independent of the filters and only depends on flux, date, and CCD position (see $\S 5.2$ ).

\subsection{MultiDrizzle}

The ACS is situated off-axis in the HST focal plane, and the ACS focal plane is not normal to incident light rays. Therefore, ACS images have a large geometric distortion (Anderson 2006;

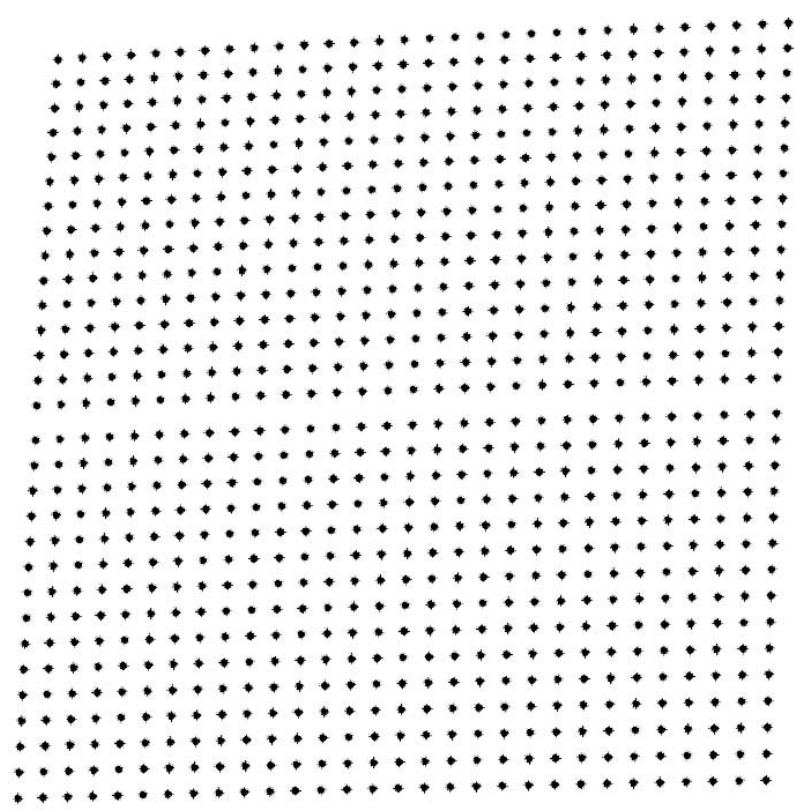

FIG. 4.- Typical artificial star field created using our modified version of Tiny Tim. This field is at a focus of $-2 \mu \mathrm{m}$.

Pavlovsky et al. 2006). The $4096 \times 4096$ ACS WFC CCDs do not sample a square on the sky; they sample a "squashed parallelogram" (see, for example, Fig. 4). The program MultiDrizzle removes this geometric distortion while simultaneously removing cosmic rays and bad pixels, as well as combining multiple exposures into a single output image (Koekemoer et al. 2002). The geometric distortion is easily measured and corrected for with astrometric observations of stellar fields, as shown by Meurer et al. (2002) and Anderson (2006). They fit the geometric distortion to a quartic polynomial, and the resulting fit is good to better than 0.05 pixels across the ACS WFC. Subsequent observations have improved this fit, and the latest distortion polynomial coefficients are readily available from the Space Telescope Science Institute. Despite the fact that the geometric distortion is the largest PSF effect, it is the easiest to correct for, and we use the default correction available via MultiDrizzle. The geometric distortion is not completely temporally stable, and indeed has changed over the lifetime of the ACS (Anderson 2006). However, the size of this variation is much smaller than the variation in PSF due to changes in the telescope's focus. The latest geometric distortion corrections are used in the reduction of the COSMOS data (Koekemoer et al. 2007). The pixel scale of the MultiDrizzle output image can be smaller than the pixel scale of the input image (or images). The details of how MultiDrizzle is run on COSMOS data are given by Koekemoer et al. (2007). We make some important changes in the default MultiDrizzle parameters in order to optimize the images for weak-lensing analysis as described in $\S 3$.

\subsection{Tiny Tim}

There are not enough stars in each extragalactic COSMOS ACS image to allow us to model the PSF across the field through interpolation. Therefore, we have simulated artificial stars in ACS fields at arbitrary positions (in the following discussion we will use the terms "star" and "PSF" interchangeably) using the Tiny Tim software package (Krist \& Hook 2004). Tiny Tim can create PSFs for any current HST camera with any filter combination, at any detector position, and for any given input spectrum. Tiny Tim creates FITS images containing stars that include the effects 
of diffraction, geometric distortion, and charge diffusion within the CCDs. By default, the stars appear as they would in raw HST images. In the case of ACS, this means the stars are highly distorted and have a pixel scale of $0.05^{\prime \prime} \mathrm{pixel}^{-1}$. Tiny Tim is able to make highly oversampled PSFs and can incorporate the focus position (primary-to-secondary mirror spacing of the telescope).

We have adapted version 6.3 of the Tiny Tim software package to create simulated ACS star fields. By default, ACS creates only single stars or several stars in a small portion of the ACS WFC. Using the IDL programming language, we have written a wrapper that allows us to run Tiny Tim multiple times and create a grid of PSF models across the whole ACS field of view. This is available online. ${ }^{12}$ We insert our artificial stars into blank images with the same dimensions and FITS structure as real ACS data, thereby manufacturing arbitrarily dense star fields. The default Tiny Tim pipeline calculates a diffraction pattern (spot diagram), geometrically distorts this pattern, and adds charge diffusion between adjacent pixels. The geometric distortion coefficients built into Tiny Tim were current at the time of the program's release but have since been superseded. Our IDL Tiny Tim wrapper allows us to input the most recent distortion coefficients. These three effects occur (in that order) in real data and depend on the position of the star in the ACS field of view. Tiny Tim is run in steps, and, with our modifications, the Tiny Tim software allows some of these steps to be skipped. By skipping various steps within our IDL wrapper we allow for two important changes to this basic pipeline. The deviations from the default Tiny Tim pipeline are as follows:

1. In order to examine the effects of the distortion removal process (MultiDrizzle in our case), we incorporate the option to allow each star to have an identical diffraction pattern and charge diffusion, but a geometric distortion determined by the location of the PSF within the ACS field of view. Once the geometric distortion is removed by running the field through MultiDrizzle, these stars should all appear identical.

2. We allow Tiny Tim to create star fields that do not contain the effects of geometric distortion at all, instead modeling stars as they would appear after a perfect removal of geometric distortion. Conversion between distorted and nondistorted frames, which is necessary to simulate charge diffusion in the raw CCD, is performed using very highly oversampled images. This avoids stochastic aliasing of the PSF (see $\S 3$ ) and minimizes noise in the PSF models. This makes use of the geometric distortion coefficients described in Gonzaga et al. (2005).

Our modified version of Tiny Tim thus allows us to make dense artificial star fields over a range of focus values. We have created star fields in the F814W filter for the COSMOS analysis, but the procedures we have developed are general and can be adapted to any filter. For reasons of speed, we ray-trace through the optical path at a single wavelength of $814 \mathrm{~nm}$. Using a full stellar (or galaxy) spectrum in the F814W filter does not significantly change the output PSF but adds considerably to the processing time. We generate the PSF models in an oversampled pixel frame. This can be repixelized to the ACS pixel size in geometrically distorted (raw) coordinates or in geometrically corrected coordinates. In the distorted coordinates, we find that the simulated PSF is slightly smaller than for real stars, possibly because of pixelization effects. This is fixed by convolving the PSF model with a square kernel the same size as the pixels. Note that, as discussed in $\S 2.1$, our Tiny Tim PSF models do not include all PSF effects, especially near the red end of the spectrum.

\footnotetext{
12 See http://www.astro.caltech.edu/ rjm/acs.
}

We also have included the ability to create a set of raw images with an arbitrary dither pattern. Stars can be added to each geometrically distorted dithered image in such a way that all the stars line up with each other on the output image. This portion of our Tiny Tim pipeline makes use of the wtranback coordinate transformation routine built into PyRAF. We can then make four dithered images with, for instance, the COSMOS dither pattern and then combine them into a single output file using MultiDrizzle (see, for example, Fig. 5). This is nontrivial because the large geometric distortion in ACS can substantially change the relative spacing of stars in dithered images. We describe the results of such tests in the following section.

\subsection{Shape Measurement}

Here we introduce our formalism for the measurement of galaxy shapes. The method of Rhodes et al. (2000, hereafter RRG) has been optimized for space-based images with small PSFs and has previously been used for weak-lensing analyses of WFPC2 and STIS data (Rhodes et al. 2001, 2004; Refregier et al. 2002). Following the formalism of RRG, we parameterize object shapes by measuring the Gaussian-weighted second-order moments:

$$
I_{i j}=\frac{\sum w I x_{i} x_{j}}{\sum w I} .
$$

The summation is over all pixels, $w$ is the size of the Gaussian weight function, $I$ is the pixel intensity, and the coordinates $x_{i}$ are measured in pixels. These moments are used to derive the ellipticity of an object $e_{i}$ and a size $d$, given by

$$
\begin{aligned}
& e_{1}=\frac{I_{x x}-I_{y y}}{I_{x x}+I_{y y}}, \\
& e_{2}=\frac{2 I_{x y}}{I_{x x}+I_{y y}}, \\
& d=\sqrt{\frac{I_{x x}+I_{y y}}{2} .}
\end{aligned}
$$

The RRG method also requires the measurement of the five fourthorder moments, given by

$$
I_{i j k l}=\frac{\sum w I x_{i} x_{j} x_{k} x_{l}}{\sum w I},
$$

in order to correct for the effects of the weighting function and the shape distortions caused by the PSF. These fourth-order moments are used to make small corrections to the second-order moments. In this way, the RRG method is a bridge between the earlier KSB (Kaiser et al. 1995) method, which uses only secondorder moments, and more advanced methods such as shapelets (Massey \& Refregier 2005), which uses moments to arbitrarily high order based on the amount of information available in each object.

The ellipticities are related to the shear, the quantity of interest for weak lensing, via the shear susceptibility factor $G$ :

$$
\gamma_{i}=\frac{e_{i}}{G}
$$

Previous weak-lensing analyses using the RRG method have made use of a single value of $G$ for the entire survey. We find that residual PSF systematics are reduced when $G$ is allowed to vary as a function of object size $d$, ellipticity $e$, and $\mathrm{S} / \mathrm{N}$. Leauthaud 

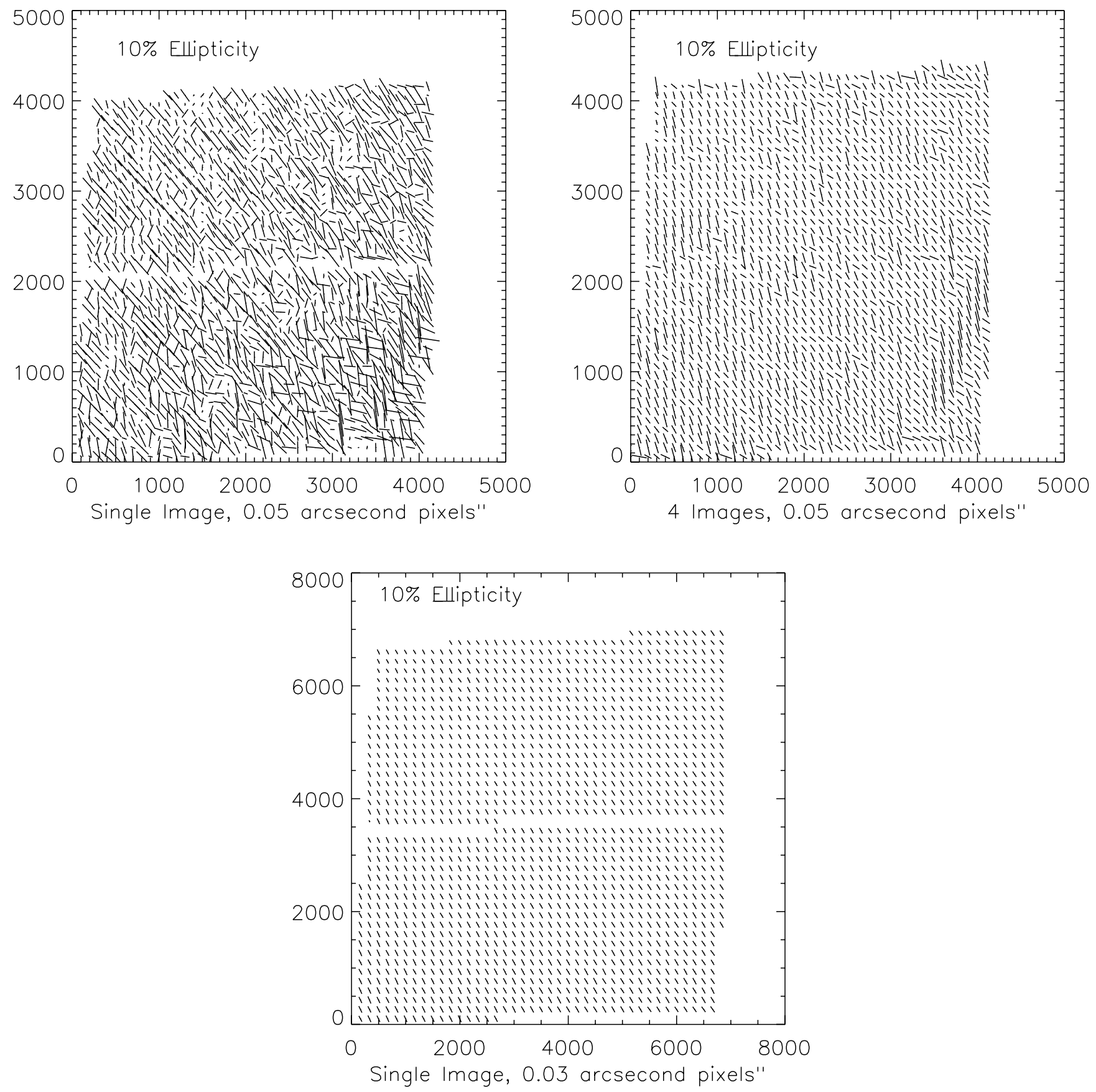

FIG. 5.-Aliasing of the PSF introduced when transforming distorted input images to an undistorted output frame. Each tick represents the ellipticity of a star that has undergone identical diffraction in the telescope's optics. Each tick mark should therefore look identical. The difference between stars is the subpixel position when geometric distortion is removed via the MultiDrizzle program. The top left panel shows a single image run through MultiDrizzle with the default settings. This problem can be ameliorated by altering several of the MultiDrizzle settings and using dithered input images. The top right panel shows that the ellipticity scatter is reduced when four dithered images are combined with the default MultiDrizzle settings. We show in Fig. 7 and in the bottom panel that reducing the output pixel scale greatly reduces this source of PSF uncertainty. This panel shows the results when a single input image is processed using our optimized MultiDrizzle settings, including a final output pixel scale of 0.03 ". The scatter in the output ellipticities is further reduced by using four dithered images with optimized MultiDrizzle settings. We do not show this plot, as the reduced ellipticity scatter is difficult to detect by eye. The interchip gap visible in the top left and bottom panels is covered by the dither pattern, which was one of the goals of the COSMOS dither pattern.

et al. (2007) contains a discussion of the calculation of $G$ for the COSMOS data set. Given an output pixel size of $0.03^{\prime \prime}$ (see $\S 3$ ) and the SExtractor parameters in Leauthaud et al. (2007), we find that the optimal weight function size for COSMOS data is

$$
w=\max (2 \sqrt{a b}, 6),
$$

where $a$ and $b$ are the SExtractor -computed semimajor and semiminor axes, and the minimum weight-function width, 6 , has been empirically determined to be the optimal width to measure the shapes of stars. These parameters (especially the factor of 2 in eq. [7]) have been tuned empirically and depend on the SExtractor settings.

\section{OPTIMAL IMAGE REDUCTION}

The transformation of pixels from a distorted input image to an undistorted output plane can introduce significant aliasing of pixels if the output pixel scale is comparable in size to the input 


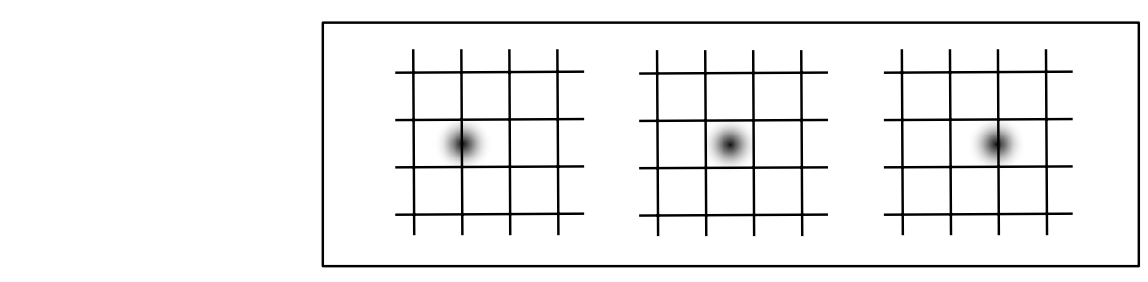

A first pixelization at the focal plane is unavoidable

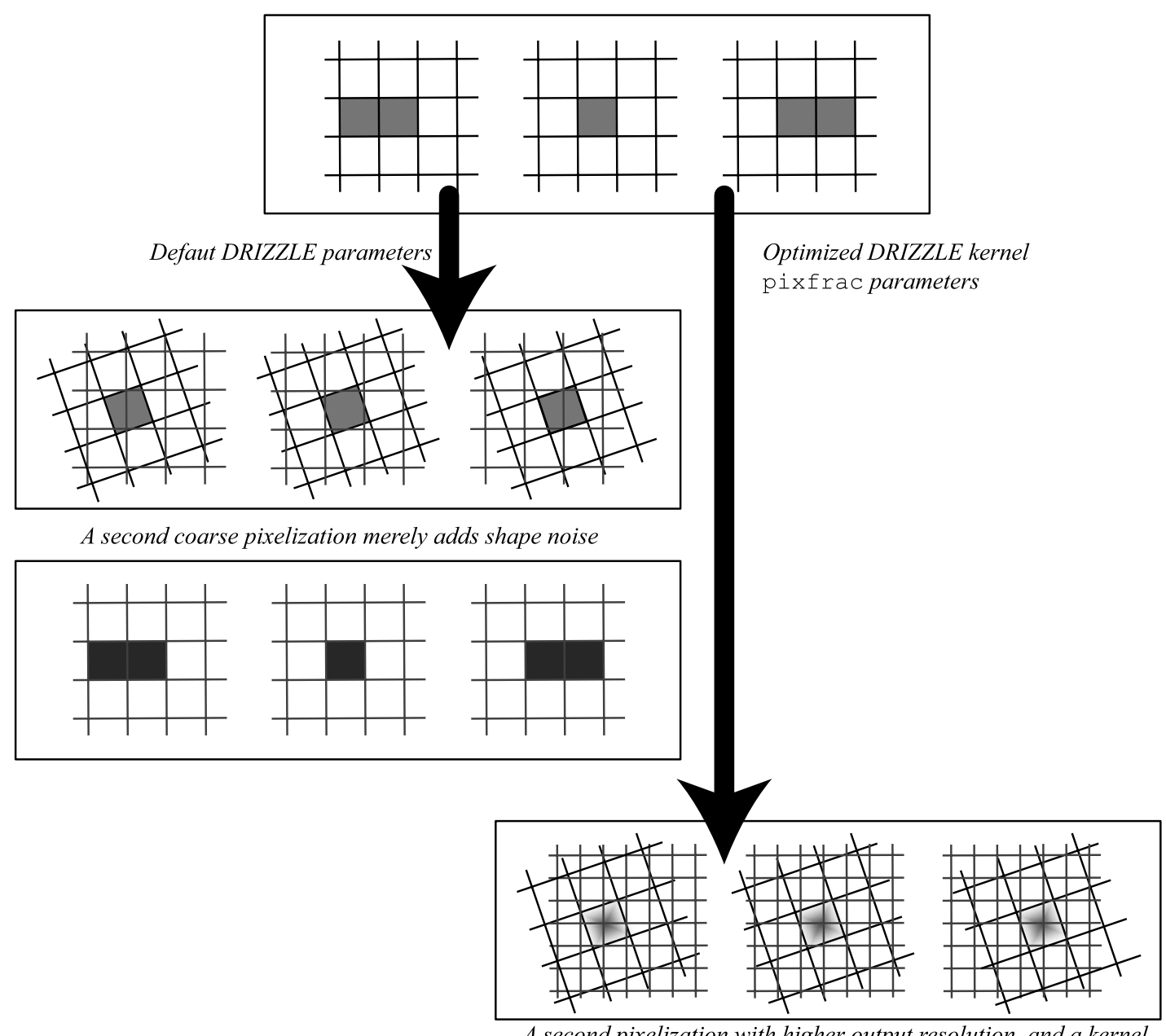

A second pixelization with higher output resolution, and a kernel that better preserves the action of a PSF as a pure convolution
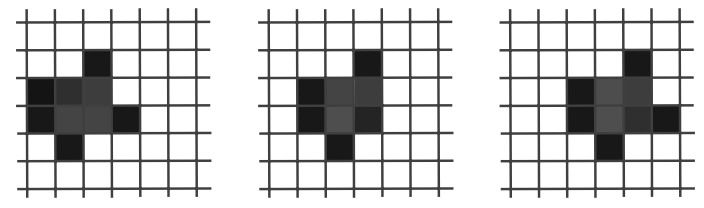

FIG. 6.-Aliasing of images caused by pixelization. The first aliasing effect (top two rows) is an inevitable consequence of observing the continuous sky with discrete pixels. The top row shows a simulated object on a pixel grid, and the second row shows how that object would appear in a pixelized image. Point sources that lie near the center of a pixel are mostly detected in that pixel, as shown in the center column. However, if the center of an object lies near the border between two pixels, the object is elongated in one direction during the process of observation. Such an elongation mimics the shear caused by weak lensing and is shown in the left and right columns of the first two rows. The middle two rows illustrate a second pixelization that can change the shapes of observed objects. This pixelization occurs during the MultiDrizzle stage of image processing, when images are resampled onto a new output grid in order to remove geometric distortion. In each of the three instances here, a perfectly centered star in the original (black tilted) coordinate frame is drizzled onto the underlying output pixel grid, which is aligned with the page. A second aliasing occurs at this stage if the input pixel lies on the border of two output pixels. Again, the middle column shows no aliasing, but the left and right images show the object being elongated in one direction. The image distortion in the second pixelization is, however, somewhat avoidable. If the output pixel grid is made smaller, as shown in the bottom two rows, the shape of the object on the final pixel grid is much less dependent on the relative alignments of the input and output pixels. Subpixel dithering of many input images and subsequent image combination onto a fine output pixel grid can further reduce the effects of pixelization. [See the electronic edition of the Journal for a color version of this figure.] 
scale. When transforming a single input image to the output plane, point sources can be enlarged and their ellipticities changed significantly depending upon their subpixel position. This is one of the fundamental reasons why dithering is recommended for observations, since the source is at a different subpixel position in different exposures, thus partially mitigating these effects when exposures are combined. However, the residual aliasing in combined images is sufficient to prevent the measurement of small, faint galaxies at the precision required for weak-lensing analysis.

In order to demonstrate the effects of aliasing we created a set of Tiny Tim star fields that contain the same diffraction and diffusion for each star but a geometric distortion given by the position of the star within the ACS WFC field. We then used MultiDrizzle (with default settings) to remove this geometric distortion. The resulting undistorted field should contain stars that are identical in all portions of the chip. We show in the top left panel of Figure 5 that this is not the case. In this figure, each tick mark represents a Tiny Tim-created star. The length and orientation of the tick mark represents the size and direction of the star's ellipticity as given by equations (2) and (3). The fact that the tick marks vary is evidence of aliasing. We have found that dithering and reducing the size of the output pixels reduces the scatter in the ellipticities of stars in this study. The largest gain comes from reducing the size of the output pixels, but, as expected, the scatter in $e$ also drops as roughly $\sqrt{N}$ for $N$ dithers. This confirms the idea that the repixelization adds stochastic noise to the ellipticity when the subpixel positions are uncorrelated. Thus, subpixel aliasing is not a problem when there are many dithers (e.g., the Hubble Ultra Deep Field), but for fields with a limited number of dithers like COSMOS, this is a significant source of PSF error. As we show below, this effect can be minimized by carefully choosing several MultiDrizzle parameters.

Pixelization effects are unavoidable during the initial exposure, when the detector discretely samples an image. However, it is clearly desirable to minimize related effects during data reduction. The effect on each individual object depends on how the input and output pixel grids line up. These arguments are demonstrated graphically in Figure 6. That figure demonstrates that we can mitigate this effect by using a finer grid of output pixels (e.g., Lombardi et al. 2005). This reduction in pixel scale (which will cause a corresponding increase in computer overheads) can be performed in conjunction with simultaneously "shrinking" the area of the input pixels that contains the signal, by making use of the MultiDrizzle pixfrac parameter and convolution kernel.

We have run a series of tests on the simulated PSF grids to determine the optimal values of the MultiDrizzle parameters specifically for weak-lensing science. As described above, we produced a grid of stars that ought to look identical after the removal of geometric distortion. We then ran a series of tests using MultiDrizzle on the same input image but with a range of output pixel scales, convolution kernels, and values of pixfrac. We measured the scatter in the ellipticity values in the output images. The smaller the scatter, the more accurately the PSF is represented. We found the results were not strongly dependent on the choice of pixfrac and settled on pixfrac $=0.8$. We show in Figure 7 that PSF stability is improved dramatically by reducing the output pixel scale from $0.05^{\prime \prime}$ (the default) to $0.03^{\prime \prime}$. There is a very slight gain in going to smaller output pixel sizes, but the storage requirements increase rapidly as the number of output pixels is increased. The gain in going to smaller pixel scales is more stable with a Gaussian kernel than with the default square kernel. Therefore, for weak lensing we use an output pixel scale of $0.03^{\prime \prime}$, pixfrac $=0.8$, and a Gaussian kernel. Despite its clear advan-

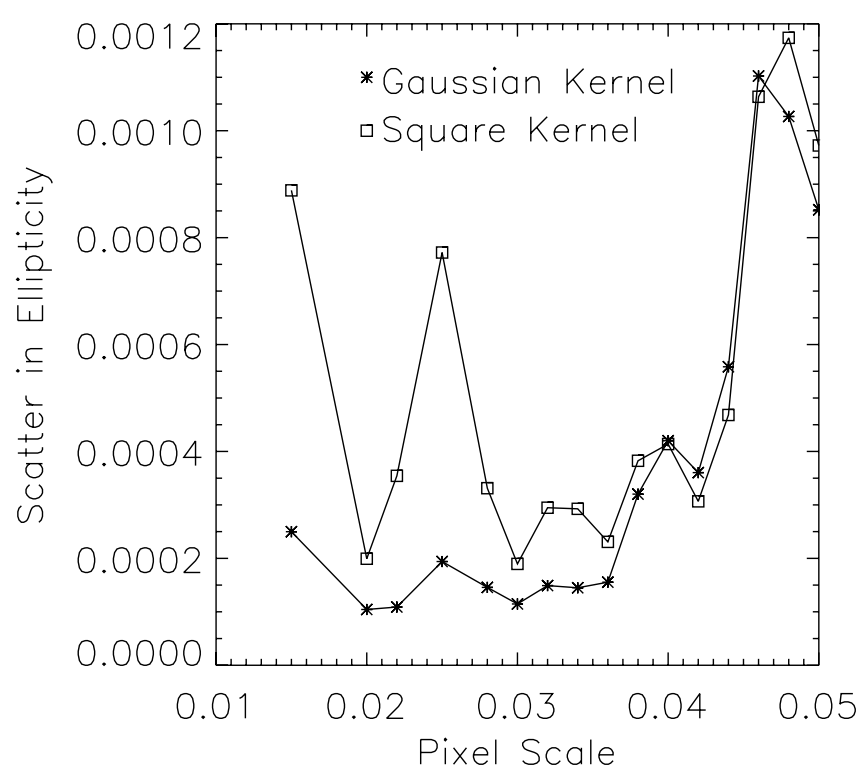

FIG. 7.- The rms ellipticity introduced during MultiDrizzle. Lower values of the scatter in ellipticity show more stable behavior of the PSF during this stage of image combination and geometric distortion removal. The only sources of $e$-variation here are pixelization and measurement errors; each star was designed to have the same PSF after the removal of geometric distortion. Each measurement represents a grid of $20 \times 20$ stars spread across the ACS WFC field. The scatter in the ellipticity is measured as the standard deviation of the mean of measured ellipticity values. Note the resonance in the square kernel at $0.025^{\prime \prime}$ pixels, half the original pixel size. The reduction of output pixel scale is the most important improvement we make on the default MultiDrizzle pipeline, with a slight further gain from going to a Gaussian rather than square kernel. For the COSMOS weak-lensing images, we use a Gaussian kernel and an output pixel size of $0.03^{\prime \prime}$ (Leauthaud et al. 2007). This minimizes the effects of undersampling on the PSF and produces images that are optimal for weak-lensing science at the cost of introducing significant additional correlated noise relative to the square kernel. This is not critical for weak-lensing science but may not be optimal for other types of science.

tages for weak-lensing studies, the Gaussian kernel does have some general drawbacks, such as the introduction of more correlated noise, which may not be desirable for other types of science where minimization of correlated noise is important.

\section{FOCUS-DEPENDENT PSF MODELS}

Changes in the HST's focus significantly change the PSF. These focus changes are caused by a change in the primary-to-secondary mirror spacing brought about by thermal fluctuations. This spacing (which we hereafter refer to as the focus) can deviate from its nominal value in the range -10 to $+5 \mu \mathrm{m}$. We thus want to know what the PSF looks like at each point in the ACS WFC field for this entire range of focus values. The repixelization of ACS data necessary to remove geometric distortion causes stochastic aliasing of the PSF, even with the optimal MultiDrizzle parameters presented in $\S 3$. Because of this, we create Tiny Tim stellar fields without geometric distortion. These contain stars that have diffraction and diffusion given by their position within the ACS WFC field, but appear as they would after a perfect removal of geometric distortion. These are stars as they would appear if the number of exposures $N$ were very large. Since we are creating simulated stars, we make the spacing between stars small enough that we do not have to do a complicated interpolation between stars to find the PSF at a particular point in the ACS WFC field. We simply choose the closest star. We find that this condition is satisfied if we create grids of $30 \times 30$ stars across the two ACS WFC CCDs. We also find that creating such a grid in the focus 

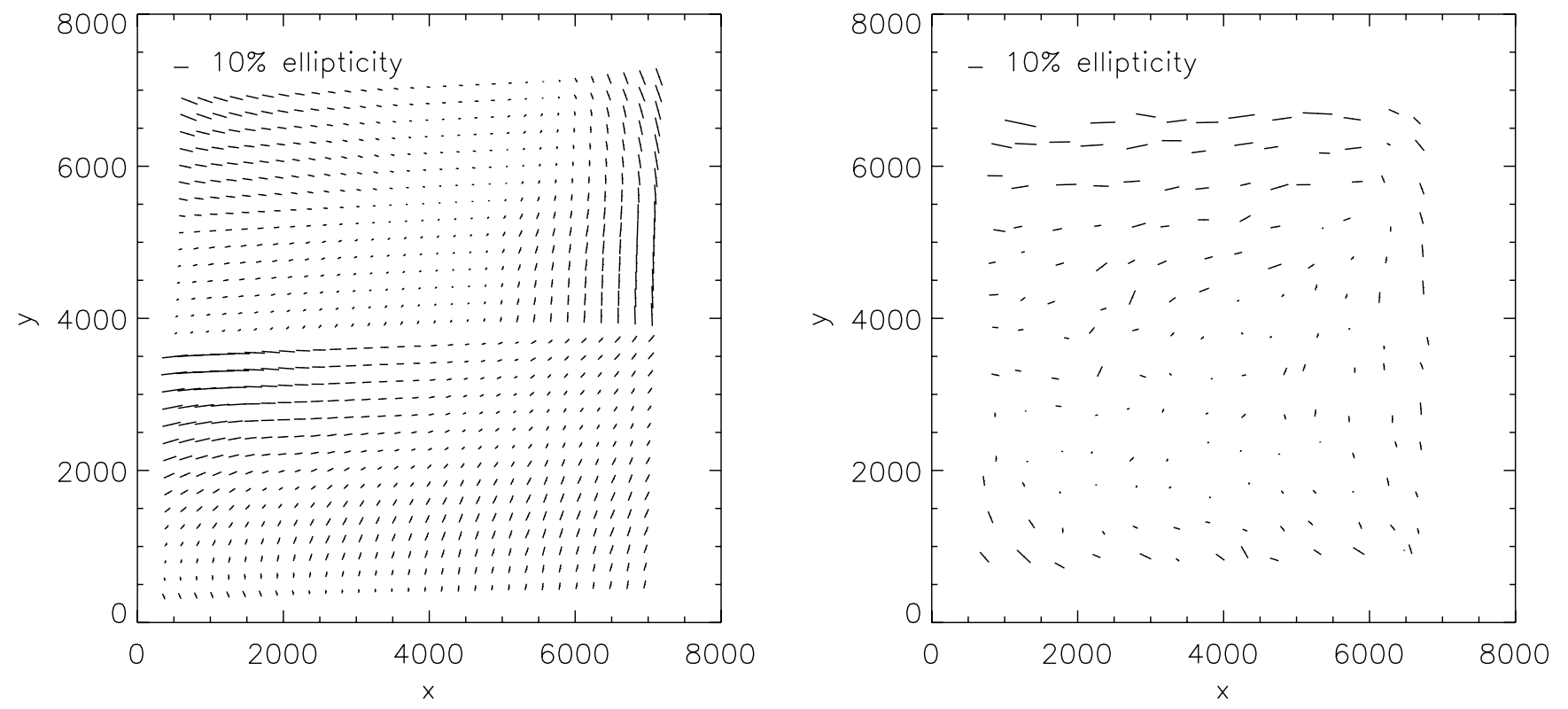

FIG. 8. - Tiny Tim PSF model (left) for a focus value of $-2 \mu \mathrm{m}$ and the average of many observed stars (right) from COSMOS fields with a similar apparent focus. There is rough qualitative agreement between the data and the models over much of the ACS field. The center of the chip does not show good agreement due, at least in part, to the effects of degradation of the CTE. The real data show less positive $e_{1}$ (elongation along the $x$-axis) than the models, consistent with a CTE-induced smearing in the $y$-direction. We remove this instrumental signature late in our lensing analysis as described in $\S 5.2$ Notice that the $x$ - and $y$-ranges are not $[0,4096]$ as in default ACS images but are larger because we are using a smaller output pixel scale. These plots are not meant to demonstrative quantitative agreement between the models and the real stars. The Tiny Tim models are noiseless, while the real COSMOS stars have image noise, and each tick mark represents a different number of stars (usually about 10 or fewer). For the RRG method, it is important for the Tiny Tim stars to match the moments (rather than the more easily plotted ellipticities) of the real stars. A quantitative analysis of how well the model stars can be used to deconvolve the PSF can be obtained from the star-galaxy correlation functions in Fig. 15 and in Massey et al. (2007a).

range -10 to $+5 \mu \mathrm{m}$ at $1 \mu \mathrm{m}$ increments gives sufficient resolution in focus that the difference in ellipticity between a star at two adjacent focus values (at the same chip position) is smaller than the measurement error in measuring the ellipticity of a star in a typical noisy COSMOS image.

In a typical COSMOS field approximately 10 stars have a suitable $\mathrm{S} / \mathrm{N}$ and are sufficiently deblended from other objects to provide accurate PSF measurements. See Leauthaud et al. (2007) for a description of star-galaxy separation in the COSMOS weaklensing catalog. We compare these stars to each focus model in the range $[-10 \mu \mathrm{m}, 5 \mu \mathrm{m}]$. We determine the Tiny Tim focal position that minimizes the $\chi^{2}$ between the ellipticities of the stars in the data and the corresponding closest stars in the Tiny Tim model, where the $\chi^{2}$ is defined as

$$
\chi^{2}=\sum\left(e_{1}^{*}-e_{1}^{\mathrm{TT}}\right)^{2}+\left(e_{2}^{*}-e_{2}^{\mathrm{TT}}\right)^{2} .
$$

The asterisk and the TT superscript represent real COSMOS stars and Tiny Tim-simulated stars, respectively, and the sum is over all the stars in the image that make the cuts described above. Thus, we determine the best fit for the telescope's focus at the time of the observation. This is the focus value we show in Figure 2. Note that this method of determining focus necessarily averages over any intraorbit focus changes. Figure 8 shows the stellar ellipticity pattern in the ACS WFC for a value of focus equal to $-2 \mu \mathrm{m}$ alongside the pattern formed by averaging all of the approximately 2000 COSMOS stars determined to be at that focus value. There is fair qualitative agreement between the data and the model. This agreement is poor in the center of the ACS WFC field. We have determined that part of this disagreement is due to CTE degradation, which is not included in the Tiny Tim PSF models. We discuss the causes of this and our solution in $\S 5.2$. The Tiny Tim model shown represents the best match to the ACS COSMOS data from all the focus values for which we calculated
Tiny Tim focus models. The real test of the quality of our PSF models is in the star-galaxy correlation functions before and after PSF correction. These are shown in Figure 15.

We have tested our focal position determination using randomly selected stars taken from the calibration images of 47 Tuc described in $\S 2.1$. We select 20 random nonoverlapping sets of 20 stars from the 746 stars seen in one of the 47 Tuc images. We determine the focus for each of these sets of stars. As these stars are all from the same image, the focal position determined should be identical for all sets. We find that all of the determined focal positions are within $\pm 2 \mu \mathrm{m}$ of the mean focal position. Based on these simulations, the results of which are shown in Figure 9, we estimate the uncertainty with which we can measure the focal position in a short exposure to be about $\pm 1 \mu \mathrm{m}$. The uncertainty in determining the focus value for a COSMOS image is somewhat larger than this because we average over the intraorbit focus changes caused by telescope breathing (see Fig. 2).

\section{PSF CORRECTION IN COSMOS IMAGES}

We follow the procedure given in RRG to correct galaxy shapes for the PSF. Stellar moments are used to correct galaxy moments first for the isotropic portion of the PSF, then for the anisotropic portion. Quantities are kept in terms of moments (rather than ellipticities) through the entire correction process, and only then are the corrected moments used to calculate galaxy ellipticities and, ultimately, shear. We tested using a variable stellar weight function width $w$ instead of the standard (for RRG) fixed stellar weight function width and found that this did not significantly improve the PSF correction. The weight function used to evaluate galaxies still varies with the size of the galaxy as discussed in $\S 2.4$.

\subsection{Applying the Tiny Tim Models}

For each COSMOS field, we choose the best-fit focus value for the telescope as described above. For each galaxy in that field 


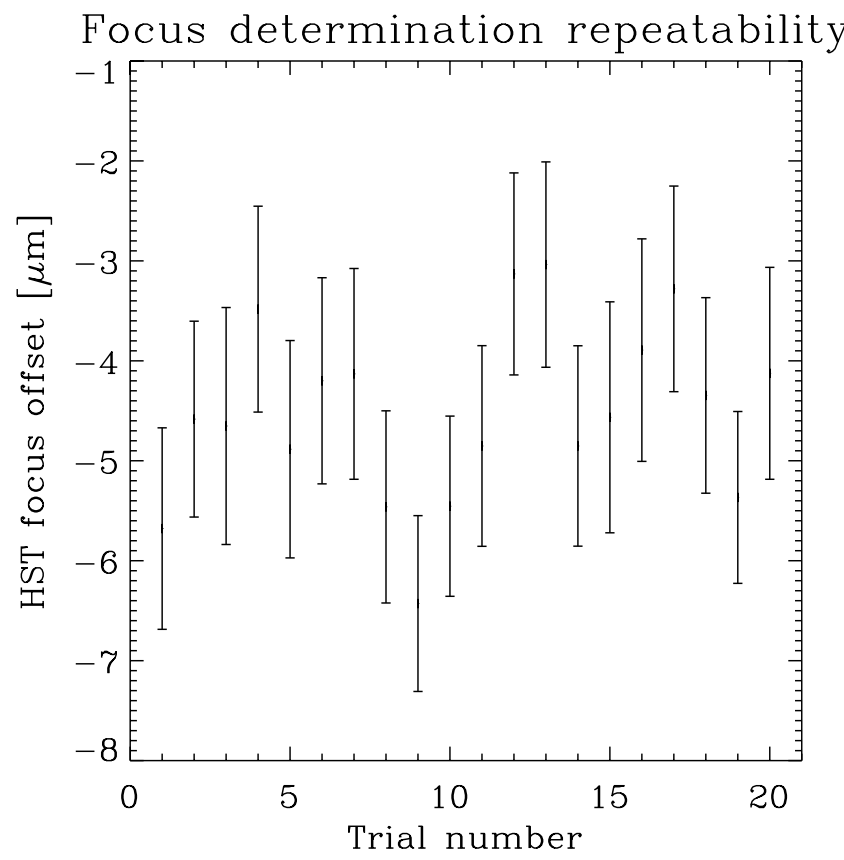

FIG. 9.-Determination of the focal position from 20 sets of 20 independent stars, each chosen randomly from a single image taken of a stellar field near the globular cluster 47 Tuc. The $x$-axis shows the set number, from 1 to 20 , and the $y$-axis shows the determined focal position. The uncertainty on the $y$-axis is the measured uncertainty of the focal position. As is shown, the focal position determination is highly repeatable in random trials of independent stars at identical focal positions. From these sorts of tests, we estimate that the uncertainty in determining the focal position for a short exposure is approximately $\pm 1 \mu \mathrm{m}$. The determination of the focus from a set of exposures taken over a full orbit has a greater uncertainty due to the intraorbit "breathing" of the telescope.

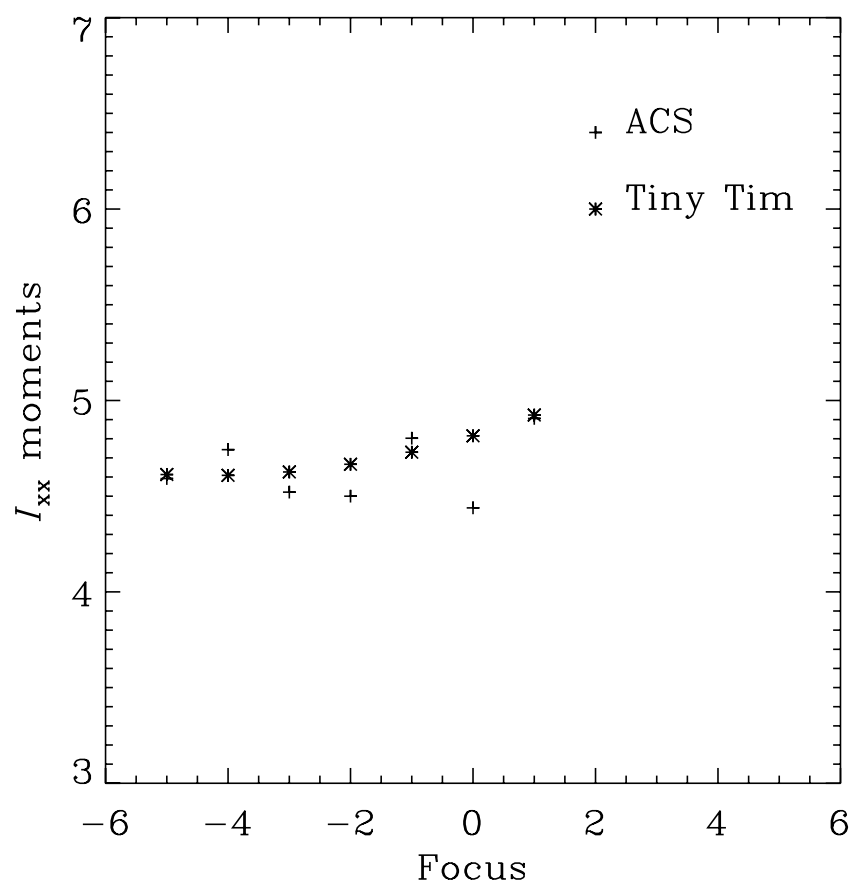

we correct the measured galaxy moments for the PSF effects with the Tiny Tim model star at that focus which is closest to the galaxy's position. We have obviated the need to interpolate the PSF across the field by creating sufficiently dense grids of model PSFs. We have found that the Tiny Tim models match the ellipticities (and more importantly the second-order moments) of the COSMOS stars they are meant to represent. However, the Tiny Tim models do not have the exact profile of the real COSMOS stars, and thus the fourth-order moments are somewhat too small in the Tiny Tim models. Only the moments with even powers of both $x$ and $y$ are affected. We show, for a range of focus values, the average of $I_{x x}$ and the average of $I_{x x x x}$ for both the Tiny Tim models and the COSMOS stars in Figure 10. The fourth-order moments are used only as a perturbative correction to the second-order moments in the RRG method, so this slight discrepancy between model and real stars does not significantly affect our PSF correction. We verify this by multiplying the Tiny Tim fourth-order moments by a correction factor and rerunning our PSF correction routines, and we find no difference in residual PSF systematics. For completeness, we tabulate the multiplicative factor between the Tiny Tim and COSMOS stars in Table 1. It is unclear why the Tiny Tim models have fourth-order moments that are slightly too small, but it may have to do with how the charge diffusion kernel is applied. Another option for PSF correction is to use the Tiny Tim model stars for focus determination and then to create interpolated PSF models using real COSMOS stars at each focus position. After acceptance of this paper, and as described in Massey et al. (2007a), this was found to slightly reduce residual PSF systematics when implemented on the COSMOS data.

\subsection{Correction for Charge Transfer Efficiency Degradation}

Gradual damage to CCD detectors due to exposure to charged particle radiation in the harsh environment of space results in a degradation in the efficiency of charge transfer in the CCDs. Highenergy charged-particle hits create charge traps that accumulate in

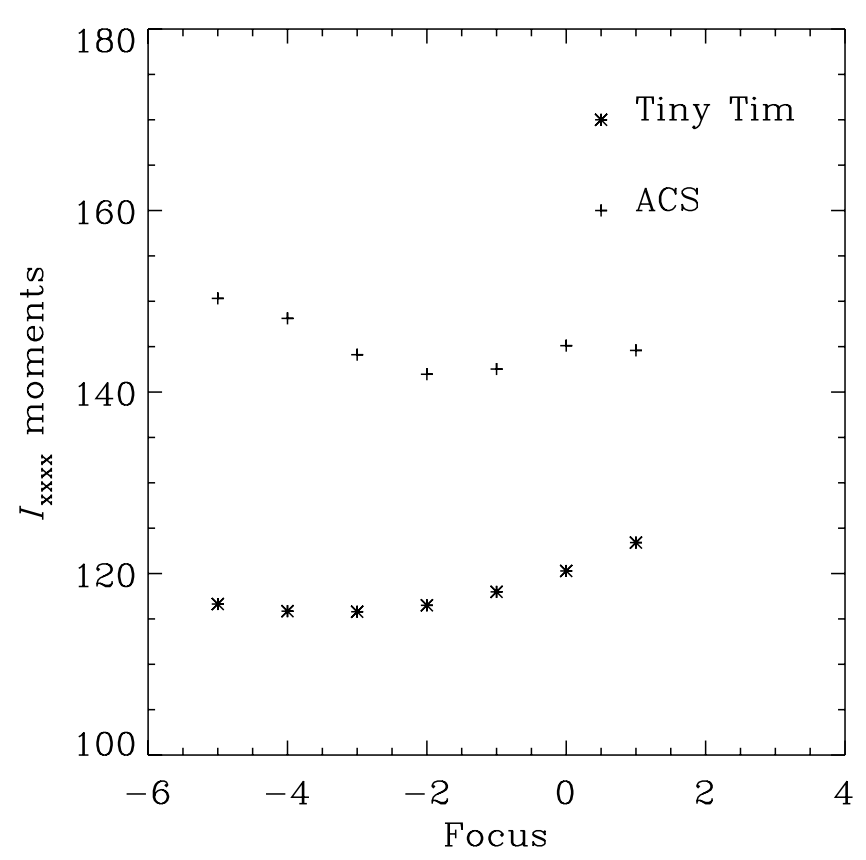

FIG. 10.- Left panel shows the values of the $I_{x x}$ moments for COSMOS stars and Tiny Tim model stars as a function of telescope focus values. There is good agreement between the two. However, the right panel shows that the Tiny Tim models are not perfect. The fourth-order moment $I_{x x x x}$ is consistently underestimated by Tiny Tim. The correction factors between the real and Tiny Tim moments are tabulated in Table 1. 
TABLE 1

Multiplicative Conversion between Tiny Tim and Measured Moments

\begin{tabular}{|c|c|}
\hline Moment & Conversion Factor \\
\hline$I_{x x}$ & 1 \\
\hline$I_{y y} \ldots \ldots \ldots \ldots \ldots \ldots \ldots \ldots$ & 1 \\
\hline$I_{x y} \ldots \ldots \ldots \ldots \ldots \ldots$ & 1 \\
\hline 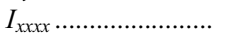 & 1.2 \\
\hline$I_{x x y} \ldots \ldots \ldots \ldots \ldots \ldots \ldots$ & 1 \\
\hline$I_{x x y y} \ldots \ldots \ldots \ldots \ldots \ldots$ & 1.1 \\
\hline$I_{x y y y} \ldots \ldots \ldots \ldots \ldots \ldots \ldots \ldots \ldots \ldots \ldots$ & 1 \\
\hline 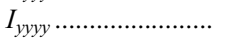 & 1.2 \\
\hline
\end{tabular}

Notes.-Multiplicative factor between the size of moments in the Tiny Tim models and the COSMOS stars. This is the factor that the Tiny Tim models would have to be multiplied by to have them equal in size to the moments of the COSMOS stars.

the silicon substrate. These traps capture electrons for a short time, and release them after a time delay drawn from an exponential distribution. Several species of traps can exist, each with its own exponential time constant. When an image is read out after exposure, the electrons from sources are stepped across these charge traps. During their capture and subsequent release, the electrons trail away from the original sources, across the image in the direction opposite to the readout. This spreading of the charge in the readout direction creates problems for photometry, astrometry, and galaxy shape measurement. Significant effort has already gone into understanding the CTE of the ACS WFC (Mutchler \& Sirianni 2005) and correcting photometric errors due to charge loss (Riess \& Mack 2004). However, the subtle changes in the shapes of objects due to CTE degradation have not yet been adequately addressed.

Since ACS was installed in 2002, charge traps have accumulated to the level of tens per pixel. There are at least three different trap species, each with different release times for trapped electrons (M. Sirianni 2006, private communication). On the two-CCD WFC, charge readout occurs at the top of the top chip and the bottom of the bottom chip; charge is read out from the center of the field to the top and bottom edges. Charge that is incident farthest from the readout registers is transferred over more pixels and therefore interacts with more traps during its translation to the chip edge. The effect on the shape of any one galaxy is difficult to predict, as it nonlinearly depends on the galaxy's magnitude, size, ellipticity, and radial profile, as well as its position on the CCD. Furthermore, the fixed number density of charge traps has the consequence of affecting faint sources more than bright ones; 100 delayed electrons are significant in a source containing 1000 electrons, but not in one containing 100,000. Thus, the shapes of distant galaxies are smeared by this effect, but less so the bright stars that are typically used to calibrate and test the galaxy shape measurement algorithms necessary for weak lensing. Thus, CTE degradation is a particularly difficult systematic effect to correct, because it cannot be calibrated using bright, high-S/N stars as is typically done with other systematics that affect weak-lensing measurements.

The effect of the CTE degradation is a smearing of objects in the readout $(y)$ direction. For faint galaxies, the size of this effect is comparable to the size of the weak-lensing signal we are trying to detect, as shown in Figure 11. The smearing is caused by each pixel leaving an exponential trail during readout, as shown in Figure 12. We have developed preliminary models for what CTE

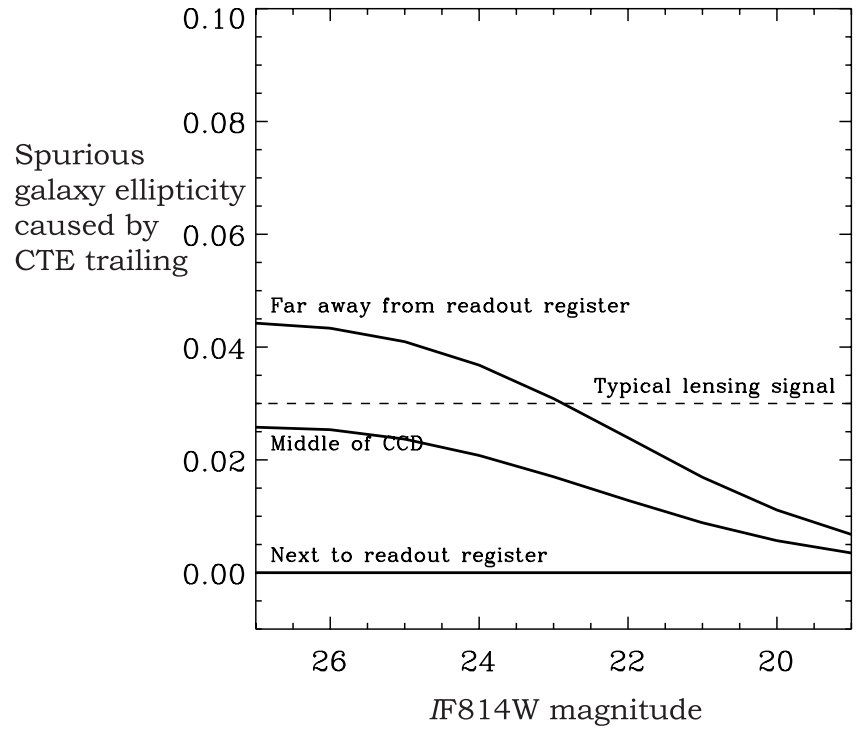

FIG. 11.-Estimate of the spurious ellipticity induced by CTE charge trailing in a barely resolved circular galaxy as a function of galaxy flux and at various positions on the CCD. In practice, the actual amount of spurious ellipticity also depends on the intrinsic ellipticity and the radial profile of the object.

degradation does to the charge in individual pixels, and we use that to show the effect of CTE degradation on the image of a faint galaxy in Figure 13.

Previous weak-lensing work with $H S T$ has encountered similar problems. Rhodes et al. (2004) corrected for CTE in STIS by creating $\mathrm{S} / \mathrm{N}$-dependent PSF models. That is impractical here because we are already dealing with focus-dependent PSF models, and the computing power and complexity required to model stars across the ACS WFC field at a range of focus values and S/N values would quickly become prohibitive. Furthermore, the CTE degradation grows worse with time, and the COSMOS images

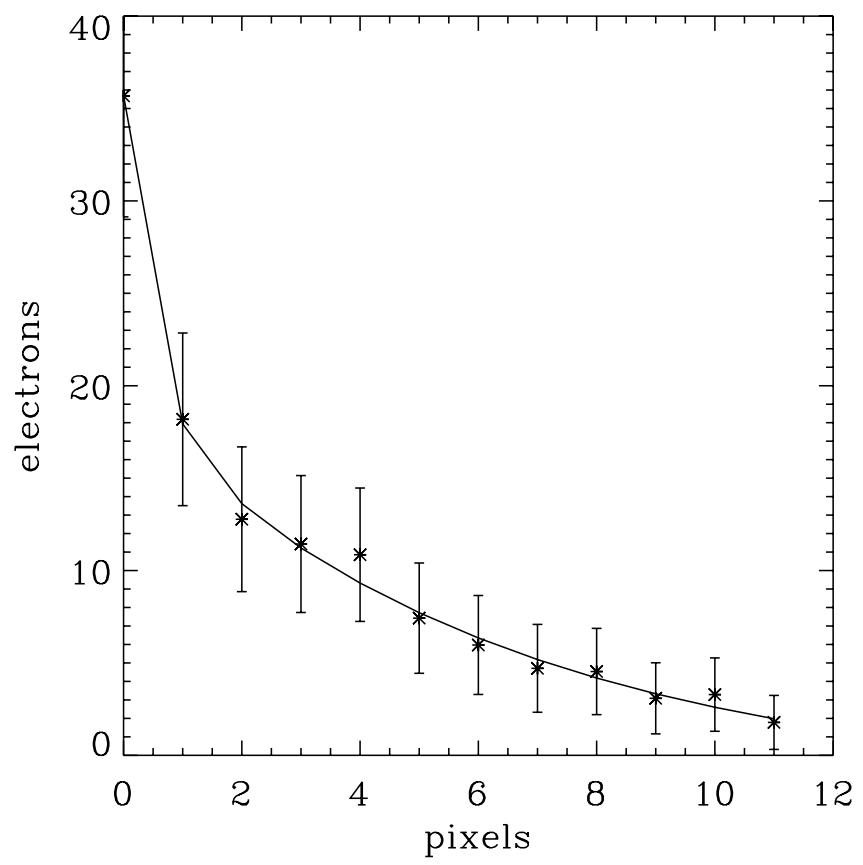

FIG. 12.-Exponential profile created during charge readout as measured in an EPER image as described in $\S 2.1$. The value of the pixel that this tail corresponds to is about 2500 electrons. 

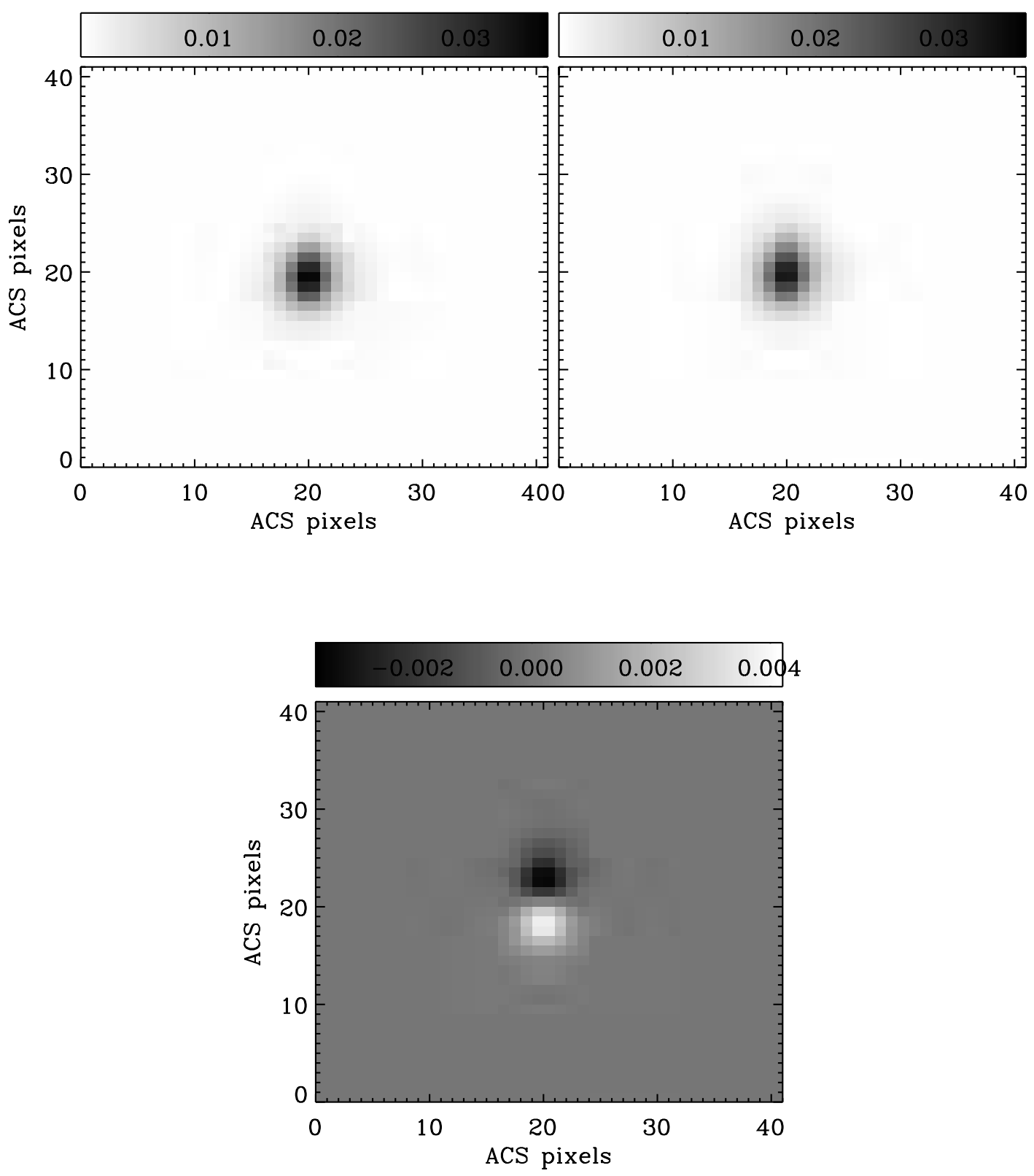

FIG. 13. - Illustration of the effect of charge trailing on a 25 mag star in single-orbit F814W data, using a crude pixel-level CTE model we have derived. The readout direction is down. A simulated galaxy is shown in the top left panel as it ought to appear and, in the top right panel, after being read out from the furthest side of the CCD. The difference between the images is small and cannot be detected by eye in the images shown. The difference is, however, significant at the level of precision needed for weak lensing. This difference is shown in the bottom panel (with a greatly expanded gray scale). We eventually hope to understand how charge is transferred between individual pixels in the ACS CCD. For this work we correct for the overall effect the CTE has on galaxy ellipticities with eq. (10).

are taken over a relatively long time period. Ideally, we would correct each pixel of each image for the effects of CTE degradation as the first step in the image reduction pipeline (see, for example, Bristow et al. 2002). CTE effects are the last to go into the image, since they occur during the readout. However, this would require an exact knowledge of the number of charge traps and the number of species of charge traps (there are at least three species, each with different release times), and accurate knowledge of the release times of those charge traps. We are working on a general solution to this problem that incorporates all of that knowledge. In the meantime, we have developed a parametric equation that allows us to correct the measured ellipticities of galaxies for the effects of CTE degradation. The CTE depends on the position of the object within the CCD (electrons farther from the readout registers encounter more charge traps), the flux of the object (high-flux objects fill the charge traps, and the relative loss offlux is less), and the date of observation (the CTE is continually degrading due to cosmic-ray damage). By assuming that the PSF-corrected ellipticities of all the galaxies in the COSMOS fields average to zero, Massey et al. (2007a) have empirically found the dependence of CTE effects on these three variables, and we use this empirical knowledge to derive the parametric correction equation

$$
e_{1}^{c}=e_{1}^{M}-e_{\mathrm{CTE}}
$$

where $e_{1}^{c}$ is the CTE-corrected first ellipticity component in the undistorted image coordinate system (which is changed by only a few degrees from the distorted coordinates), and $e_{1}^{M}$ is the measured first ellipticity component after correction for other PSF effects using the RRG method, as described above. Only the first 

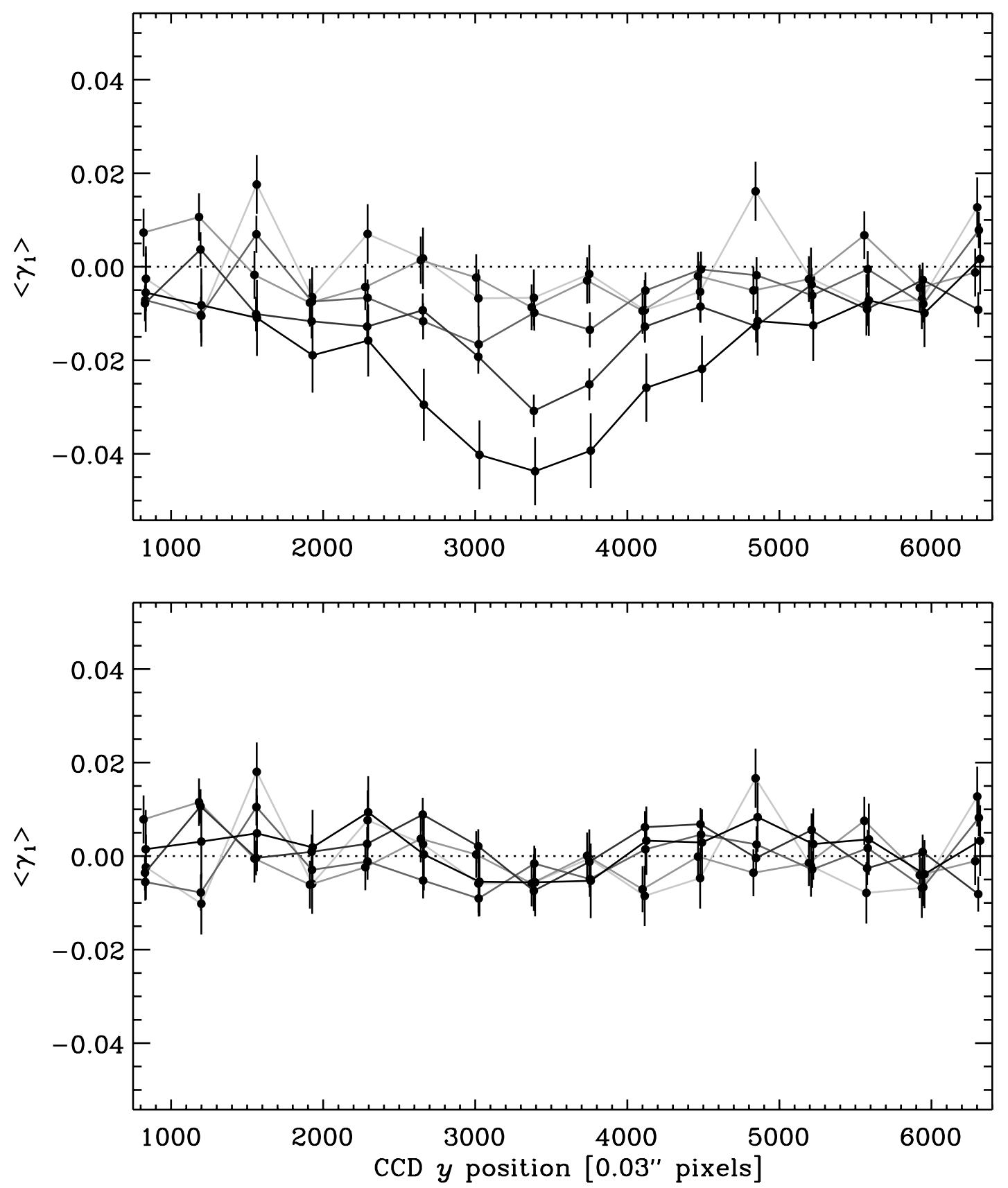

FIG. 14.- Top panel shows the average ellipticity as a function of $y$-position for several magnitude ranges after the RRG PSF correction scheme. The lowest line in the top plot represents galaxies in the magnitude range 26-27. The other lines represent $1 \mathrm{mag}$ bins going up to 22-23. The effects of CTE are clearly shown by the fact that fainter objects (with lower flux) are preferentially elongated in the $y$-direction, corresponding to negative $e_{1}$. The problem is worst near the center of the field, farthest from the readout registers. The bottom panel shows that a simple additive factor (as given by eq. [10]) on the ellipticity of each galaxy removes the CTE-induced ellipticity. In the future we hope to understand the CTE well enough to remove CTE effects from the images before a lensing analysis.

ellipticity component is affected, because this component represents elongation in the $x$ - and $y$-direction. The term $e_{2}$ represents elongation along axes at $\pm 45^{\circ}$ from the $x$-axis. The additive correction parameter $e_{\mathrm{CTE}}$ is given by

$$
e_{\mathrm{CTE}}=-3 \times 10^{-5}(\mathrm{~S} / \mathrm{N})^{-1}\left(\frac{n_{\text {tran }}}{2048}\right)(\mathrm{MJD}-52,333),
$$

where $\mathrm{S} / \mathrm{N}$ is the object's detection signal-to-noise ratio, $n_{\text {tran }}$ is the distance (number of transfers) to the nearest readout register in native ACS pixels, and MJD is the modified Julian date of the observation. Note that this empirically derived correction depends on date, $y$-position (number of readouts), and flux, just as the CTE effect is known to. We were able to obtain similar results by adjusting the exponent on the $\mathrm{S} / \mathrm{N}$ term while adding a sizedependent term. However, we choose this formalism because it is simpler and more physically motivated. It is important to note that this particular formalism for the CTE is only valid on data taken before 2006 July. At this time the operating temperature of the ACS WFC CCDs was changed, thus changing the CTE of the CCDs (Sirianni et al. 2006).

We show in Figure 14 the ellipticities of galaxies as a function of $y$-position and magnitude both before and after the CTE correction for the entire COSMOS survey. The telltale $\operatorname{dip}$ in $e_{1}^{M}$ found in the data, implying an elongation in the $y$-direction, is removed by this parametric CTE correction. Before correction, the mean 


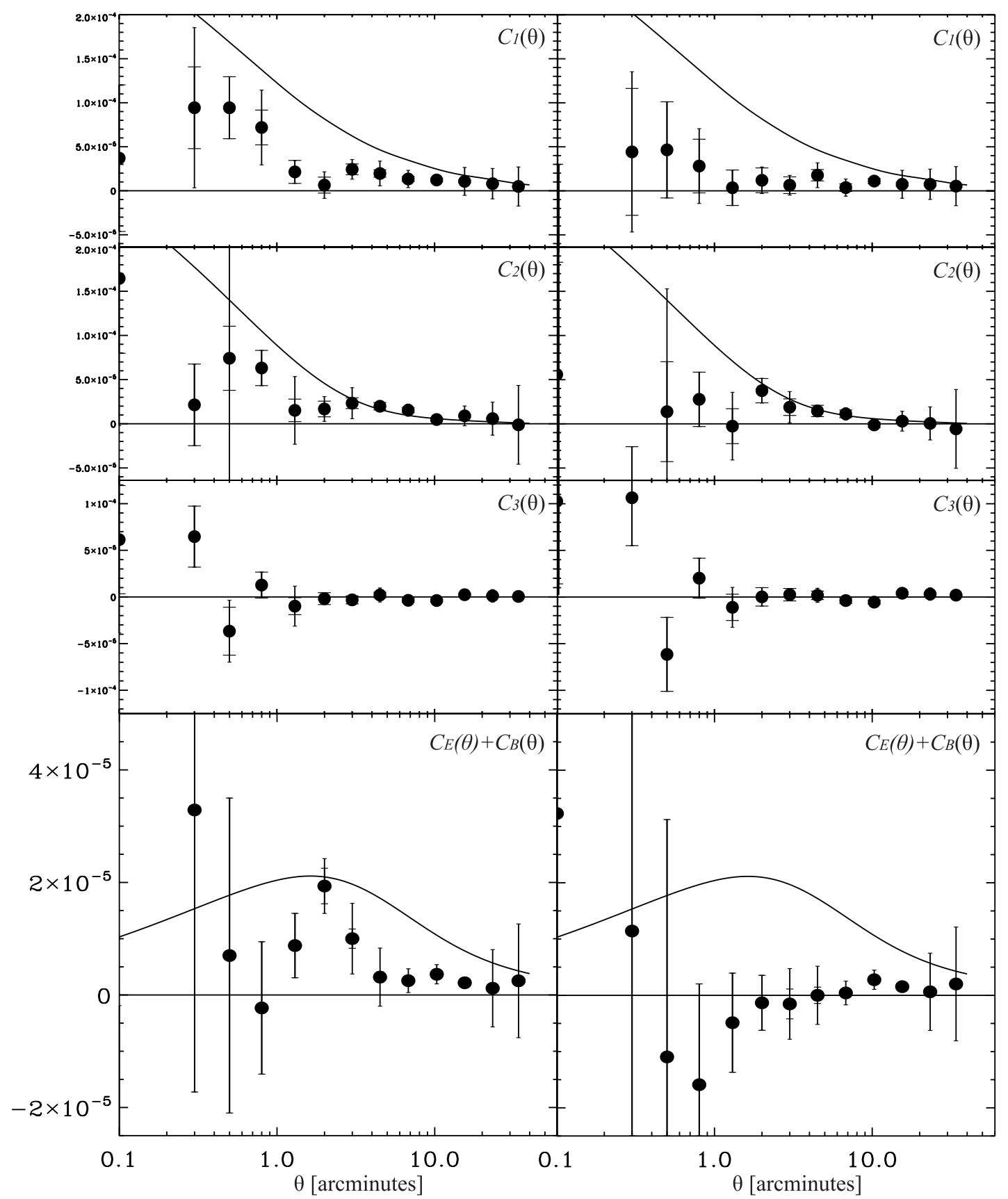

FIG. 15. - Correlation functions between galaxy shears and raw stellar ellipticities, showing the contamination of the shear catalog by residual PSF artifacts. The inner error bars contain statistical errors only, and the outer error bars include the variation found by dividing the COSMOS field into four quadrants and performing the correlation function analyses on each quadrant independently. The left panels show the shear measured straight from the images; the right panels show the shear after correction. The solid lines show the best-fitting cosmic shear signal measured by Massey et al. (2007a) for comparison. The success of our correction scheme is demonstrated by the lowering of the data points to be consistent with zero after correction, especially in the plots of the $E$ - and $B$-mode signal.

ellipticity of the whole galaxy population is $\left\langle e_{1}^{M}\right\rangle=-0.020 \pm$ 0.001 , and the gradient of a fit to the faintest magnitude bin in Figure 14 is $\partial e_{1} / \partial n_{\text {tran }}=(-1.74 \pm 1.3) \times 10^{-4}$. After correction, these values are reduced to $\left\langle e_{1}^{c}\right\rangle=0.004 \pm 0.002$ and $\partial e_{1} / \partial n_{\text {tran }}=$ $(-1.53 \pm 1.0) \times 10^{-5}$. Note that in two-point shear correlation functions (e.g., Massey et al. 2007a) this residual enters only as the value squared. In the sense of that statistic, we have therefore lessened the impact of CTE trailing in faint galaxies by more than 2 orders of magnitude.

Despite its apparent success, we stress that this prescription is by no means a panacea for CTE effects in ACS weak-lensing data. In this simplified model, we take advantage of the uniform back- ground level of the COSMOS images to eliminate dependence on this parameter. The other model parameters are also specific to our data set and shear measurement method. An improved, pixel-level CTE correction method, along the lines of Bristow et al. (2002), will follow (R. J. Massey \& J. D. Rhodes 2007, in preparation). This method will take into account the different species of charge traps and their associated release times.

\subsection{Performance of the PSF Correction}

To demonstrate the correction of the ACS data for PSF and CTE degradation, we show correlation functions between the galaxy shears and the raw stellar ellipticities in Figure 15. Stars and 
galaxies should be correlated before PSF correction (due to convolution by the same PSF) but should be uncorrelated after PSF correction. Note that in the RRG method we do not correct the stars for PSF convolution. Thus, after PSF correction the stars should contain the PSF signal, but the galaxies should not, and their ellipticities should be uncorrelated. Further descriptions of the use of correlation functions in weak lensing can be found in, for instance, Bacon et al. (2003) and Kamionkowski et al. (1998). This figure shows

$$
\begin{gathered}
C_{1}(\theta)=\frac{\sigma_{\gamma}}{\sigma_{e^{*}}}\left\langle e_{1}^{* r}(\boldsymbol{r}) \gamma_{1}^{r}(\boldsymbol{r}+\boldsymbol{\theta})\right\rangle, \\
C_{2}(\theta)=\frac{\sigma_{\gamma}}{\sigma_{e^{*}}}\left\langle e_{2}^{* r}(\boldsymbol{r}) \gamma_{2}^{r}(\boldsymbol{r}+\boldsymbol{\theta})\right\rangle, \\
C_{3}(\theta)=\frac{\sigma_{\gamma}}{\sigma_{e^{*}}}\left[\left\langle e_{1}^{* r}(\boldsymbol{r}) \gamma_{2}^{r}(\boldsymbol{r}+\boldsymbol{\theta})\right\rangle+\left\langle e_{2}^{* r}(\boldsymbol{r}) \gamma_{1}^{r}(\boldsymbol{r}+\boldsymbol{\theta})\right\rangle\right],
\end{gathered}
$$

where $\boldsymbol{\theta}$ is the separation between a star and the galaxies, averaging is performed over the whole population, the superscript $r$ denotes components of ellipticity, and the shear is rotated so that $\hat{\gamma}_{1}^{r}\left(\hat{\gamma}_{2}^{r}\right)$ in each galaxy points along (at $45^{\circ}$ from) the vector between the pair. The normalization via star-star correlation functions suggested by Bacon et al. (2003) is impractical as a denominator in this case, because the specific PSF pattern of ACS makes it cross zero several times. Figure 15 also shows the star-galaxy correlation functions after separation into $E+B$ modes via the variance of the aperture mass statistic, as defined in Schneider et al. (2002). This figure includes error from statistics alone (inner error bars) and, when applicable, variation found by subdividing the COSMOS field into four independent quadrants and doing the analysis on each quadrant independently (outer error bars). These error bars increase in size after the PSF correction. This is due to the fact that PSF convolution circularizes objects (making their ellipticities smaller). After the PSF correction, the ellipticity distribution of objects is larger, and thus, the scatter in the correlation functions is larger.

Before correction, the shear measurements contain artifacts from the PSF anisotropy. These are largely removed by the processes described in this section, and are consistent with zero on all scales after correction. Correlation functions $C_{1}$ and $C_{2}$ show significant improvement (movement towards zero correlation), especially on scales less than $1^{\prime} ; C_{3}$ was already nearly consistent with zero before the PSF correction, and the PSF correction introduced a larger scatter due to the widening of the ellipticity distribution during the PSF deconvolution discussed in the previous paragraph. The most important test of our PSF correction is shown in the $E+B$ mode plots, which show that after the PSF correction the $E+B$ mode is consistent with zero for all scales. See Massey et al. (2007a) for a more detailed description of these correlation functions and the separation of the signal into $E+B$ modes.

\section{CONCLUSIONS}

We have shown that aliasing of the ACS WFC PSF can be minimized by carefully choosing the parameters of the image reduction pipeline element MultiDrizzle. This aliasing is minimal when MultiDrizzle is run with a Gaussian kernel, the area of the input pixels containing the flux is shrunk by a factor of pixfrac $=0.8$, and the output image has a pixel scale of $0.03^{\prime \prime}$ pixel $^{-1}$. We show that the ACS WFC PSF is temporally unstable over the timescale of the COSMOS observations due to thermally induced changes in the telescope's focus. Using a modified version of the Tiny Tim software package, we create dense
PSF grids at a range of telescope focus values from -10 to $+5 \mu \mathrm{m}$. Using the $\sim 10$ suitable stars in each image as taken from the COSMOS lensing catalog (Leauthaud et al. 2007), we can calculate the focus value of $H S T$ for each COSMOS field within about $\pm 1 \mu \mathrm{m}$. We can use the Tiny Tim models to correct the galaxy shapes for the effects of the PSF. There is a residual systematic left after the PSF correction that is due to degradation of the CTE by cosmic-ray damage to the CCDs. We present a parametric correction for the effects of CTE degradation on galaxy ellipticities. The resulting PSF- and CTE-corrected catalog has been used by Massey et al. (2007a) to measure cosmic shear.

Our wrappers and implementation of the Tiny Tim code are publicly available online. ${ }^{13}$ Since our PSF-modeling routines are general, they are applicable to other filter sets and can be used for weak-lensing data sets taken with other filters (e.g., the ACS parallel survey taken with the F775W filter or a re-reduction of the portion of the GEMS survey that was taken with the F606W filter). Similarly, the PSF models are not specific to the RRG weak-lensing method. Since we are creating simulated stars, any weak-lensing pipeline can make use of these stars for PSF deconvolution. The code and models have already been put to use in other science papers by the COSMOS collaboration in such diverse areas as AGN studies (J. Gabor et al. 2007, in preparation) and morphological classification (Scarlata et al. 2007).

Future work will include a more general correction for the effects of CTE degradation. This correction will likely take place on the images themselves as a first step in the image reduction pipeline. Given the general nature of our PSF models, we plan to use them to perform further weak-lensing analyses on the COSMOS data set with the more advanced shapelets method (Refregier 2003; Refregier \& Bacon 2003; Massey \& Refregier 2005).

We thank Stefano Casertano and Andy Fruchter for useful discussions on the ACS PSF and how it is affected by drizzle. Our use of the Tiny Tim software would not have been possible without the help of John Krist. The HST COSMOS Treasury program was supported through NASA grant HST-GO-09822. We wish to thank Tony Roman, Denise Taylor, and David Soderblom for their assistance in the planning and scheduling of the extensive COSMOS observations. We gratefully acknowledge the contributions of the entire COSMOS collaboration, consisting of more than 70 scientists, and of the COSMOS PI Nick Scoville in particular. More information on the COSMOS survey is available at http://www.astro.caltech.edu/cosmos. It is a pleasure to acknowledge the excellent services provided by the NASA/IPAC Infrared Science Archive staff(Anastasia Laity, Anastasia Alexov, Bruce Berriman, and John Good) in providing online archive and server capabilities for the COSMOS data sets. The COSMOS Science meeting in 2005 May was supported in part by the NSF through grant OISE-0456439. We thank the anonymous referee for useful comments that improved the paper immensely. We also thank Matthew Lallo and Russell Makidon for providing focus data for the HST during the time the COSMOS observations were made. R. M. was supported in part by grant HST-AR-10964. This research was carried out in part at the Jet Propulsion Laboratory, California Institute of Technology, under a contract with NASA and finded through the internal Research and Technology Development program.

\section{Facilities: HST (ACS)}

\footnotetext{
13 See http://www.astro.caltech.edu/ rjm/acs.
} 
Anderson, Jay 2006, in Proc. 2005 HST Calibration Workshop: Hubble after the Transition to Two-Gyro Mode, ed. A. Koekemoer, P. Goudfrooij, \& L. Dressel (NASA CP-214134; Washington: NASA), 11

Bacon, D., Massey, R., Refregier, A., \& Ellis, R. 2003, MNRAS, 344, 673

Bristow, P., et al. 2002, in Proc. 2002 HST Calibration Workshop: Hubble after the Installation of the ACS and the NICMOS Cooling System, ed. S. Arribas, A. Koekemoer, \& B. Whitmore (Baltimore: STScI), 176

Gonzaga, S., et al. 2005, ACS Instrument Handbook, Ver. 6.0 (Baltimore: STScI)

Heymans, C., et al. 2005, MNRAS, 361, 160 2006, MNRAS, 368, 1323

Hoekstra, H., Frenx, M., Kuijken, K., \& Squires, G. 1998, ApJ, 504, 636

Jee, M., et al. 2006, ApJ, 642, 720 2005, ApJ, 634, 813

Kaiser N., Squires G., \& Broadhurst T. 1995, ApJ, 449, 460

Kamionkowski, M., et al. 1998, MNRAS, 301, 1064

Koekemoer, A., Fruchter, A., Hook, R. \& Hack, W. 2002, in Proc. 2002 HST Calibration Workshop: Hubble after the Installation of the ACS and the NICMOS Cooling System, ed. S. Arribas, A. Koekemoer, \& B. Whitmore (Baltimore: STScI), 337

Koekemoer, A., et al. 2007, ApJS, 172, 196

Krist, J. 2003, ACS WFC and HRC Field-Dependent PSF Variations Due to Optical and Charge Diffusion Effects (ACS-ISR 2003-06; Baltimore: STScI)

Krist, J., \& Hook, R. 2004, The Tiny Tim User's Manual (Baltimore: STScI)

Lallo, M., et al. 2005, HST Temporal Optical Behavior and Current Focus Status (ISR TEL 05-03; Baltimore: STScI) 2006, Proc. SPIE, 6270, 54

Leauthaud, A., et al. 2007, ApJS, 172, 219

Lombardi, M., et al. 2005, ApJ, 623, L42

Makidon, R., et al. 2006a, in Proc. 2005 HST Calibration Workshop, ed. A. Koekemoer, P. Goudfrooij, \& L. Dressel (NASA CP-214134; Washington: NASA), 405

\section{FERENCES}

Makidon, R., et al. 2006b, Proc. SPIE, 6270, 52

Massey, R. J., \& Refregier, A. 2005, MNRAS, 363, 197

Massey, R. J., et al. 2007a, ApJS, 172, 239

. 2007b, MNRAS, 376, 13

2007c, Nature, 445, 286

Meurer, G., et al. 2002, in Proc. 2002 HST Calibration Workshop: Hubble after the Installation of the ACS and the NICMOS Cooling System, ed. S. Arribas, A. Koekemoer, \& B. Whitmore (Baltimore: STScI), 65

Mutchler, M., \& Sirianni, M. 2005, Internal Monitoring of ACS Charge Transfer Efficiency (ISR ACS 05-03; Baltimore: STScI)

Pavlovsky, C., et al. 2006, ACS Instrument Handbook, Ver. 7.0 (Baltimore: STScI)

Refregier, A. 2003, MNRAS, 338, 35

Refregier, A., \& Bacon, D. 2003, MNRAS, 338, 48

Refregier, A., Rhodes, J., \& Groth E. J. 2002, ApJ, 572, L131

Rhodes, J.D., Refregier, A., \& Groth, E. J. 2000, ApJ, 536, 79

- 2001, ApJ, 552, L85

Rhodes, J. D., et al. 2004, ApJ, 605, 29 2006, in Proc. 2005 HST Calibration Workshop: Hubble after the Transition to Two-Gyro Mode, ed. A. Koekemoer, P. Goudfrooij, \& L. Dressel (NASA CP-214134; Washington: NASA), 21

Riess, A., \& Mack, J. 2004, Time Dependence of ACS WFC CTE Corrections for Photometry and Future Predictions (ISR ACS 04-06; Baltimore: STScI) Scarlata, C., et al. 2007, ApJS, 172, 406

Schneider, P., van Waerbeke, L., \& Mellier, Y. 2002, A\&A, 389, 729

Schrabback, T., et al. 2006, A\&A, submitted (astro-ph/0606611)

Scoville, N. Z., et al. 2007, ApJS, 172, 1

Sirianni, M., Gilliand, R., \& Sembach, K. 2006, The ACS Side-2 Switch: An Opportunity to Adjust the WFC CCD Temperature Setpoint (TIR 06-02; Baltimore: STScI)

Sirianni, M., et al. 2005, PASP, 117, 1049 